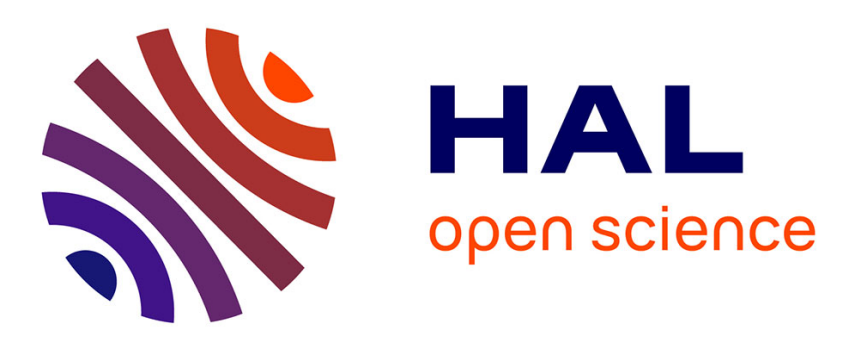

\title{
Effect of confinement in wall-bounded non-colloidal suspensions
}

\author{
Stany Gallier, Elisabeth Lemaire, Laurent Lobry, Francois Peters
}

\section{To cite this version:}

Stany Gallier, Elisabeth Lemaire, Laurent Lobry, Francois Peters. Effect of confinement in wall-bounded non-colloidal suspensions. Journal of Fluid Mechanics, 2016, 799, pp.100-127. 10.1017/jfm.2016.368 . hal-01347658

\section{HAL Id: hal-01347658 \\ https://hal.science/hal-01347658}

Submitted on 21 Jul 2016

HAL is a multi-disciplinary open access archive for the deposit and dissemination of scientific research documents, whether they are published or not. The documents may come from teaching and research institutions in France or abroad, or from public or private research centers.
L'archive ouverte pluridisciplinaire HAL, est destinée au dépôt et à la diffusion de documents scientifiques de niveau recherche, publiés ou non, émanant des établissements d'enseignement et de recherche français ou étrangers, des laboratoires publics ou privés. 


\title{
Effect of confinement in wall-bounded non-colloidal suspensions
}

\author{
STANY GALLIER ${ }^{1} \dagger$, ELISABETH LEMAIRE ${ }^{2}$, \\ LAURENT LOBR Y AND FRANCOIS PETERS \\ ${ }^{1}$ SAFRAN-Herakles, Le Bouchet Research Center, 91710 Vert le Petit, France \\ ${ }^{2}$ University of Nice, CNRS, LPMC-UMR 7336, Parc Valrose, 06100 Nice, France
}

(Received ?; revised ?; accepted ?. - To be entered by editorial office)

This paper presents three-dimensional numerical simulations of non-colloidal dense suspensions in a wall-bounded shear flow at zero Reynolds number. Simulations rely on a fictitious domain method with a detailed modelling of particle-particle and wall-particle lubrication forces, as well as contact forces including particle roughness and friction. This study emphasizes the effect of walls on the structure, velocity and rheology of a moderately confined suspension (channel gap to particle radius ratio of 20) for a volume fraction range $0.1 \leq \phi \leq 0.5$. The wall region shows particle layers with an hexagonal structure. The size of this layered zone depends on volume fraction and is only weakly affected by friction. This structure implies a wall slip which is in good accordance with empirical models. Simulations show that this wall slip can be mitigated by reducing particle roughness. For $\phi \lesssim 0.4$, wall-induced layering has a moderate impact on viscosity and second normal stress difference $N_{2}$. Conversely, it significantly alters the first normal stress difference $N_{1}$ and can result in positive $N_{1}$, in better agreement with some experiments. Friction enhances this effect, which is shown to be due to a substantial decrease in the contact normal stress $\left|\Sigma_{x x}^{c}\right|$ (where $x$ is the velocity direction) because of particle layering in the wall region.

\section{Key words:}

\section{Introduction}

Dense suspensions of particles in low Reynolds number flows are ubiquitous in industry as well as in biological or natural flows. They display complex flow properties, intermediate between solid and liquid. The present study considers the ideal case of non-Brownian non-colloidal single-sized spherical particles embedded in a Newtonian fluid. However, even this simple suspension notoriously exhibits the complex non-Newtonian behaviours typical of actual suspensions (Stickel \& Powell 2005; Morris 2009). Recent advances in understanding the complex physics of suspensions have rested on detailed experiments and numerical simulations. Simulations provide access to some micromechanical details not easily available in experiments, such as the three-dimensional microstructure, flow field or hydrodynamic and contact forces between particles. In particular, the Stokesian Dynamics (SD) (Brady \& Bossis 1988; Sierou \& Brady 2002) has been instrumental in providing insights into suspension physics. To a large extent, the current knowledge concerns unbounded suspensions since most simulations - especially SD - usually consider 
an infinite domain by using periodicity conditions in all directions.

Conversely, confined suspensions have received much less attention. Suspension flows in porous media, blood flow in capillaries or microfluidic devices are examples of situations where interactions with boundaries are predominant. This can lead to surprising effects such as a negative quadratic dependence of viscosity with volume fraction (Davit \& Peyla 2008) or swapping trajectories for two spheres in shear flow (Zurita-Gotor et al. 2007). Walls are also known to strongly alter particle diffusion (Yeo \& Maxey 2010a; Michailidou et al. 2009). Works by Sangani et al. (2011) show a significant increase in the stresslet of a particle close to a wall, by a factor of 4 . Usual rheological measurements make use of rheometers where the suspension is confined. Therefore, it is not completely known to what extent walls can alter the measured rheological quantities. There is now a debate concerning the sign of the first normal stress difference $N_{1}=\Sigma_{x x}-\Sigma_{y y}$ (where $x$ and $y$ refer to the direction of velocity and velocity gradient, respectively) since available experimental results are controversial. Numerical simulations of unbounded suspensions using Stokesian Dynamics (SD) (Sierou \& Brady 2002), Force-Coupling Method (FCM) (Yeo \& Maxey 2010b) or Fictitious Domains (Gallier et al. 2014b) find a negative $N_{1}$. Other simulations by Mari et al. (2014) show that prior to shear thickening, the average value of $N_{1}$ is nearly zero but is dominated by large fluctuations. In contrast, experiments report either negative $N_{1}$ (Zarraga et al. 2000; Singh \& Nott 2003; Dai et al. 2013), or almost zero (Couturier et al. 2011), or positive $N_{1}$ (Dbouk et al. 2013; Royer et al. 2016; Lootens et al. 2005), or, at last, either positive or negative values depending on the size and polydispersity of particles (Gamonpilas et al. 2016). Interestingly, recent simulations by Yeo \& Maxey (2010b) in wall-bounded suspensions have shown that $\left|N_{1}\right|$ was smaller than in unbounded suspensions. This suggests that confinement may have a role on rheology in general and on $N_{1}$ in particular. This is one of the motivations of the present work.

Walls are also known to affect the suspension flow field by inducing an apparent wallslip (Coussot 2005; Jana et al. 1995) which alters the effective shear in the suspension. They also promote a local ordering by forming particle layers, as confirmed by simulations (Singh \& Nott 2000; Nguyen \& Ladd 2002; Kromkamp et al. 2006; Yeo \& Maxey $2010 b$ ). This wall-induced layering is also clearly visible experimentally by high-resolution particle tracking (Blanc et al. 2013; Metzger et al. 2013; Cheng et al. 2011; Snook et al. 2015; Pieper \& Schmid 2016). An hexagonal ordering takes place close to walls and is attested for volume fractions $\phi$ as low as 0.48 (Yeo \& Maxey 2010c). This wall-induced ordering can persist on large distances, typically $10 a$ where $a$ is the particle radius. For strongly confined systems (channel width to particle radius ratio $L_{y} / a<11$ ), this order also depends on the commensurability between the channel width and the number of particle layers (Yeo \& Maxey 2010c). Simulations by Bian et al. (2014) also show that confinement increases viscosity, facilitates cluster formation and is essential to observe hydrodynamic shear thickening.

This paper intends to improve the current knowledge of the role of walls on suspensions - especially rheology - using numerical simulations. In present work, we make use of a fictitious domain approach as detailed in Gallier et al. (2014a). Our method explicitly solves long-range hydrodynamics and incorporates an adequate modelling of lubrication and contact forces. Wall-particle lubrication interactions will be specifically addressed in this work due to its relevance. The thorough study by Yeo \& Maxey (2010b) has already considered simulations on wall-bounded suspensions and has brought many significant results on the role of confinement. The present study intends to go a step further by focusing on rheology (especially normal stress differences) as well as investigating the effect of friction, which has recently been shown to have a profound impact on the rheology of homogeneous suspensions (Gallier et al. 2014b; Fernandez et al. 2013; Seto et al. 2013). 
Section 2 presents a brief description of the numerical approach used. In section 3, we present suspension simulation results in simple shear flows and investigate the role of walls on suspension structure, flow field and rheology. Throughout this study, the magnitude of confinement is described by the ratio $\kappa$ of channel width $L_{y}$ to particle radius $a$, i.e. $\kappa=L_{y} / a$. Most simulations are conducted on moderately confined suspensions $\kappa=20$.

\section{Numerical model}

This section briefly describes the numerical method used ; more details can be found in a previous paper (Gallier et al. 2014a). In a fictitious domain method, solid particles are supposed to be filled with a fluid having the same properties as the actual fluid. From a computational viewpoint, this means that a classical fluid problem is solved in the whole domain. Particles are thus considered as some regions of the fluid constrained to have a rigid body motion.

\subsection{Review of the fictitious domain method}

Particles are supposed to be rigid and homogeneous whereas the fluid is assumed incompressible and Newtonian and is governed by the Stokes equations :

$$
\begin{aligned}
\boldsymbol{\nabla} \cdot \boldsymbol{u} & =0 \\
\nabla \cdot \Sigma+\rho \boldsymbol{\lambda} & =0
\end{aligned}
$$

where $\rho$ and $\boldsymbol{u}$ are the fluid density and velocity, respectively, while $\boldsymbol{\lambda}$ is a momentum forcing term used to enforce the rigid body motion inside particles. For a Newtonian fluid, the stress tensor $\Sigma$ reads

$$
\Sigma=-p I+2 \eta E
$$

where $p$ is the pressure, $\eta$ the fluid viscosity and $E$ the rate-of-strain tensor $E=\frac{1}{2}(\boldsymbol{\nabla} \boldsymbol{u}+$ $\left.\boldsymbol{\nabla} \boldsymbol{u}^{T}\right)$. The fluid velocity inside each particle must comply with a rigid body motion, so that

$$
\boldsymbol{u}=\boldsymbol{U}+\boldsymbol{\Omega} \times\left(\boldsymbol{x}-\boldsymbol{x}_{g}\right)
$$

where $\boldsymbol{U}$ and $\boldsymbol{\Omega}$ stand for the particle translational and rotational velocities and $\boldsymbol{x}_{g}$ is the position of the centre of gravity of the particle. Particle motion is given by Newton's equations which read, neglecting inertia :

$$
\begin{aligned}
\boldsymbol{F}^{h}+\boldsymbol{F}^{c}+\boldsymbol{F}^{e} & =0 \\
\boldsymbol{T}^{h}+\boldsymbol{T}^{c}+\boldsymbol{T}^{e} & =0
\end{aligned}
$$

where forces $\boldsymbol{F}$ and torques $\boldsymbol{T}$ are decomposed into their hydrodynamic part $(h)$, contact part $(c)$ and external part $(e)$ that can include any external force, such as gravity. Stokes equations are solved by finite differences on a staggered Cartesian grid using standard projection methods. Once particle velocities are known, their position is updated using a second-order Adams-Bashforth scheme. Most numerical details are here skipped and may be found in Gallier et al. (2014a).

\subsection{Lubrication model}

Lubrication forces play a major role in concentrated suspensions and require adequate modelling. They are very short-range in nature, so that they can usually not be fully resolved with the typical grids employed. The present lubrication model is described 
elsewhere (Gallier et al. 2014a) and is here only briefly addressed. It is similar to the approach used in SD or FCM inasmuch as hydrodynamic interactions are split into longrange interactions - explicitly resolved - and short-range (lubrication) contribution that must be modelled since they can not be resolved. The grand resistance matrix $R$, that links hydrodynamic forces/torques and translational/rotational velocities, can therefore be written as

$$
R \approx R^{r}+R^{n r}
$$

The resolved part $R^{r}$ formally describes this part of interactions explicitly described by the numerical model whereas the non-resolved part $R^{n r}$ represents the contribution that can not be resolved with the actual grid. It is classically estimated by subtracting the resolved resistance matrix $R_{2 B}^{r}$ - obtained numerically on two-sphere configurations from the exact theoretical two-sphere resistance matrix $R_{2 B}^{\text {theo }}$ known from lubrication theory (Kim \& Karrila 1991). For a many-particle system, $R^{n r}$ is constructed assuming a pairwise additivity of forces. The associated non-resolved lubrication force/torque $\mathcal{F}^{n r}=$ $(\boldsymbol{F}, \boldsymbol{T})^{T}$ is related to particle velocities $\mathcal{U}=(\boldsymbol{U}, \boldsymbol{\Omega})^{T}$ by

$$
\mathcal{F}^{n r}=R_{F U}^{n r} \cdot\left(\mathcal{U}_{\infty}-\mathcal{U}\right)+R_{F E}^{n r}: E_{\infty}
$$

where $\mathcal{U}_{\infty}=\left(\boldsymbol{U}_{\infty}, \boldsymbol{\Omega}_{\infty}\right)^{T}$ and $\boldsymbol{U}_{\infty}, \boldsymbol{\Omega}_{\infty}, E_{\infty}$ are the unperturbed translational velocities, rotational velocities and rate-of-strain tensor, respectively. This force/torque $\mathcal{F}^{n r}$ represents the lubrication portion of hydrodynamic interactions that can not be resolved by the numerical approach and is directly included in Eq. (2.5)-(2.6) as an external force and torque. The hydrodynamic stresslet $S^{h}$ is corrected from lubrication as well using a similar procedure. The deviatoric stresslet is written in resistance form and is similarly decomposed into a resolved and non-resolved part as

$$
S=S^{r}+R_{S U}^{n r} \cdot\left(\mathcal{U}_{\infty}-\mathcal{U}\right)+R_{S E}^{n r}: E_{\infty}
$$

where $S^{r}$ corresponds to the resolved stresslet explicitly computed by the numerical method. The non-resolved resistance matrices $R_{S U}^{n r}$ and $R_{S E}^{n r}$ are obtained as described previously and theoretical expressions are found in Kim \& Karrila (1991). Finally, a similar correction procedure is also applied to the trace of $S^{h}$ - which represents the hydrodynamic contribution to particle pressure $\Pi$ - using the theoretical resistance functions from Jeffrey et al. (1993) and similarly reads

$$
\Pi=\Pi^{r}+R_{\Pi U}^{n r} \cdot\left(\mathcal{U}_{\infty}-\mathcal{U}\right)+R_{\Pi E}^{n r}: E_{\infty}
$$

\subsection{Lubrication near a wall}

Lubrication for wall-particle interactions are actually taken into account using a similar strategy. The non-resolved lubrication interactions are identified by subtracting off the resolved interactions (obtained on particle-wall configurations with different gaps) from the theoretical interactions. Because the latter are addressed scarcely and incompletely in the literature, they are here described in more detail. The theoretical resistance expressions for the hydrodynamic force, torque, stresslet and particle pressure for a single particle close to a wall read

$$
\left[\begin{array}{c}
\boldsymbol{F} \\
\boldsymbol{T} \\
S \\
\Pi
\end{array}\right]=\eta\left[\begin{array}{ccc}
A & B^{\dagger} & G^{\dagger} \\
B & C & H^{\dagger} \\
G & H & M \\
P & 0 & Q
\end{array}\right]\left[\begin{array}{c}
\boldsymbol{U}_{\infty}(\boldsymbol{x})-\boldsymbol{U} \\
\boldsymbol{\Omega}_{\infty}(\boldsymbol{x})-\boldsymbol{\Omega} \\
E_{\infty}
\end{array}\right]
$$


Following the notations in Kim \& Karrila (1991), the resistance tensors are

$$
\begin{aligned}
A_{i j} & =X^{A} d_{i} d_{j}+Y^{A}\left(\delta_{i j}-d_{i} d_{j}\right) \\
B_{i j} & =Y^{B} \epsilon_{i j k} d_{k} \\
C_{i j} & =X^{C} d_{i} d_{j}+Y^{C}\left(\delta_{i j}-d_{i} d_{j}\right) \\
G_{i j k} & =X^{G}\left(d_{i} d_{j}-\frac{1}{3} \delta_{i j}\right) d_{k}+Y^{G}\left(d_{i} \delta_{j k}+d_{j} \delta_{i k}-2 d_{i} d_{j} d_{k}\right) \\
H_{i j k} & =Y^{H}\left(\epsilon_{i k l} d_{l} d_{j}+\epsilon_{j k l} d_{l} d_{i}\right) \\
M_{i j k l} & =X^{M} d_{i j k l}^{(0)}+Y^{M} d_{i j k l}^{(1)}+Z^{M} d_{i j k l}^{(2)} \\
P_{i} & =X^{P} d_{i} \\
Q_{i j} & =X^{Q}\left(d_{i} d_{j}-\frac{1}{3} \delta_{i j}\right)
\end{aligned}
$$

in which $\boldsymbol{d}$ is the unit vector from the particle centre to the wall. The fourth-rank tensors $d_{i j k l}^{(0)}, d_{i j k l}^{(1)}$ and $d_{i j k l}^{(2)}$ can be found in Kim \& Karrila (1991). The third-rank transpose in Eq. (2.11) is such that $G_{i j k}^{\dagger}=G_{k i j}$.

For plane walls and a shear flow (which will be the case in this study), the functions $X^{M}, Z^{M}$ and $X^{Q}$ are actually not needed because the associated terms $d_{i j k l}^{(0)} E_{k l}, d_{i j k l}^{(2)} E_{k l}$ and $\left(d_{i} d_{j}-1 / 3 \delta_{i j}\right) E_{i j}$ are zero in that case. The required resistance functions are therefore $X^{A}, Y^{A}, Y^{B}, X^{C}, Y^{C}, X^{G}, Y^{G}, Y^{H}, Y^{M}$ and $X^{P}$. Some asymptotic near-wall developments are available only for $X^{A}, Y^{A}, Y^{B}, X^{C}, Y^{C}, Y^{G}$ and $Y^{H}$ (see Yeo \& Maxey (2010b) for a compiled set of expressions), so that $X^{G}, Y^{M}$ and $X^{P}$ seem to be missing in the literature. Since these functions are primarily connected with stresslet and particle pressure, this may confirm that the role of walls on rheology has not received much attention. In appendix A, we report the near-wall asymptotic expressions used in this work. They are taken from the literature except for $X^{G}, Y^{M}$ and $X^{P}$ for which we propose new expressions.

\subsection{Contact model}

Contact interactions are modelled using Hertzian soft-spheres. For a pair of spherical particles $i$ and $j$ (radius $a$ ) undergoing contact, the contact force $\boldsymbol{F}^{c}$ is classically decomposed into its normal $\boldsymbol{F}_{n}^{c}$ and tangential $\boldsymbol{F}_{t}^{c}$ components : $\boldsymbol{F}^{c}=\boldsymbol{F}_{n}^{c}+\boldsymbol{F}_{t}^{c}$. The normal contact force is modelled using a Hertz law

$$
\boldsymbol{F}_{n}^{c}=-k_{n}|\delta|^{3 / 2} \boldsymbol{n}
$$

in which $\delta=\|\boldsymbol{r}\|-2 a$ is the overlap distance with $\boldsymbol{r}=\boldsymbol{x}_{j}-\boldsymbol{x}_{i}$, and $\boldsymbol{n}$ is the normal vector $\boldsymbol{n}=\boldsymbol{r} /\|\boldsymbol{r}\|$. Surface roughness is accounted for in the model assuming sparse asperities of size $h_{r}$. Contact is therefore supposed to take place whenever $\|\boldsymbol{r}\| \leqslant 2 a+h_{r}$, which corresponds to define a modified overlap distance $\delta^{\prime}=\delta-h_{r}$. Hence, contact occurs if $\delta^{\prime} \leq 0$. Note that lubrication forces are however still evaluated with actual distance $\delta=\delta^{\prime}+h_{r}$ since the fluid is assumed to flow freely between asperities. In this work, the roughness size will be fixed to $h_{r} / a=5.10^{-3}$ which is a typical roughness measured for suspension particles (Smart \& Leighton 1989; Blanc et al. 2011). Walls are assumed to be perfectly smooth. The normal stiffness $k_{n}$ in Eq. (2.12) is chosen sufficiently high so as to mimic rigid particles and the non-dimensional stiffness $k_{n} / \eta \dot{\gamma} a^{2} h_{r}^{-3 / 2}$ is about $2.10^{3}$. This involves an average roughness deformation $\left|\delta^{\prime}\right| / h_{r}$ lower than 0.1 . The exact value of stiffness has been shown to induce negligible effects on rheology if 
it is sufficiently large (Gallier et al. 2014b). For dense regimes however, roughness can occasionally be deformed completely, leading to $\delta=0$. This is not numerically tractable since lubrication functions diverge at $\delta=0$. To circumvent this problem, a threshold value of $10^{-6} a$ is prescribed when evaluating lubrication functions. This means that when lubrication functions are being computed, any normalized distance $\delta / a$ lower than $10^{-6}$ is fixed to $10^{-6}$. This choice is similar to Sierou \& Brady (2001). Note that this threshold is useless and not considered when computing contact forces through Eq. (2.12).

The tangential force is given by

$$
\boldsymbol{F}_{t}^{c}=-k_{t} \Upsilon \boldsymbol{T}
$$

in which $\Upsilon$ is defined by integrating the slip velocity $\boldsymbol{U}^{s}$ during the contact duration $t_{c}$

$$
\boldsymbol{\Upsilon}=\int_{0}^{t_{c}} \boldsymbol{U}^{s} \mathrm{~d} t
$$

where the slip velocity is

$$
\boldsymbol{U}^{s}=\boldsymbol{U}_{i}-\boldsymbol{U}_{j}-\left[\left(\boldsymbol{U}_{i}-\boldsymbol{U}_{j}\right) \cdot \boldsymbol{n}\right] \cdot \boldsymbol{n}+\left(a \boldsymbol{\Omega}_{i}+a \boldsymbol{\Omega}_{j}\right) \times \boldsymbol{n}
$$

Using the classical Amontons-Coulomb law of friction, the actual tangential force magnitude is limited by the friction limit $\mu_{d}\left|\boldsymbol{F}_{n}^{c}\right|$ where $\mu_{d}$ is the dynamic friction coefficient. The tangential stiffness $k_{t}$ is linked to the normal stiffness $k_{n}$ by $k_{t} / k_{n}=2\left|\delta^{\prime}\right|^{1 / 2} / 7$ (Silbert et al. 2001; Shäfer et al. 1996). Finally, the corresponding contact torque is

$$
\boldsymbol{T}^{c}=a \boldsymbol{n} \times \boldsymbol{F}^{c}
$$

Contact forces also induce an additional contact stresslet and contact particle pressure that are given for a particle as

$$
\begin{aligned}
\boldsymbol{S}^{c} & =\frac{1}{2}\left(\boldsymbol{F}^{c} \otimes a \boldsymbol{n}+a \boldsymbol{n} \otimes \boldsymbol{F}^{c}\right) \\
\Pi^{c} & =-\frac{1}{3}\left(\boldsymbol{F}^{c} \cdot a \boldsymbol{n}\right)
\end{aligned}
$$

Contact forces with walls are handled similarly. Particle-wall interactions are frictionless if particles are themselves frictionless. Conversely, the particle-wall interaction is frictional if particle-particle friction is considered. The wall is assumed to be perfectly smooth but since particles are rough, they can experience an actual contact with the wall through particle roughness : particle-wall contacts therefore occur whenever $\delta \leqslant h_{r}$.

\subsection{Validation : single sphere near a wall}

A validation for the wall-particle hydrodynamic interactions is proposed for a single forcefree torque-free spherical particle in the vicinity of a wall in a shear flow. When a particle is close to a wall, wall-particle interactions alter particle velocity and stresslet. In particular, the wall tends to impose its own velocity to the particle and this must be accurately predicted so as to model wall-bounded suspensions. The present case considers a single particle (radius $a$ ) freely suspended in a linear shear flow of magnitude $\dot{\gamma}=2 U_{w} / L_{y}$ with $L_{y}=10 a$ is the channel width and $U_{w}$ (resp., $-U_{w}$ ) is the velocity prescribed at the upper wall (resp., lower wall) in the $x$-direction. The domain size in the velocity and vorticity direction is respectively $L_{x}=20 a$ and $L_{z}=20 a$. The grid spacing is $\Delta=a / 5$ and the time step is $\Delta t=10^{-3} \dot{\gamma}^{-1}$. Simulations are conducted for different particle vertical positions $Y$ in the channel and we note $\xi=(Y-a) / a$ the non-dimensional gap between particle surface and lower wall. The lubrication correction is activated for a non-dimensional gap lower than 0.2 . 

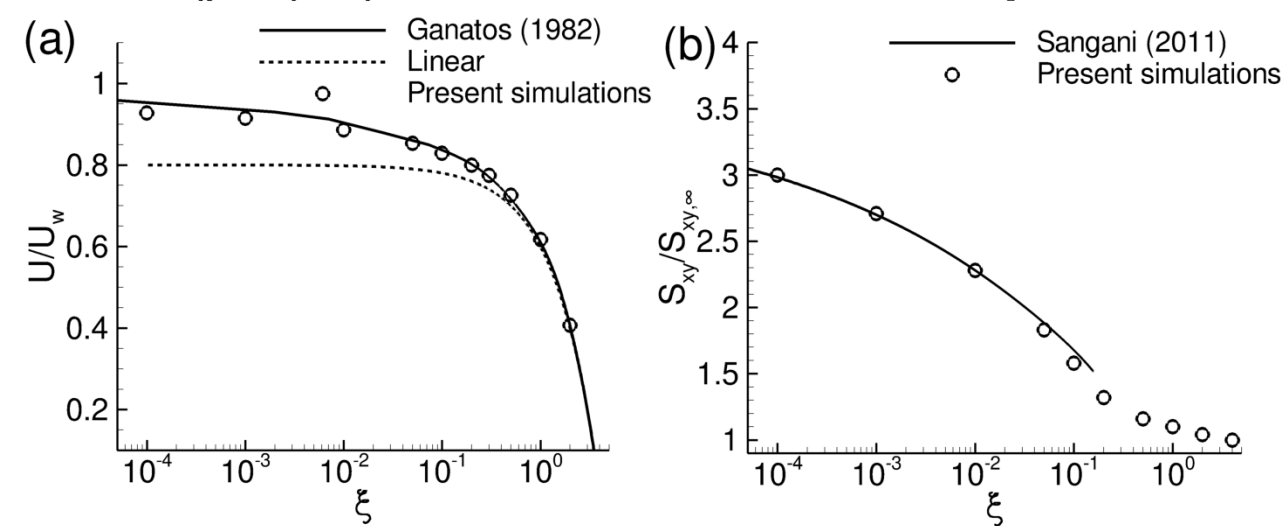

FiguRE 1. Translational particle velocity $U / U_{w}$ (a) and particle stresslet $S_{x y} / S_{x y, \infty}$ (b) in a shear flow as a function of particle non-dimensional distance to wall $\xi=(Y-a) / a$. Channel width is $L_{y}=10 a$. Solid lines in (a) : simulations by Ganatos et al. (1982). Solid lines in (b) : asymptotic expression by Sangani et al. (2011).

Figure 1(a) presents the particle translational velocity $U$ rescaled by the wall velocity $U_{w}$. Reference simulations by Ganatos et al. (1982) using a boundary collocation method are also reported. Results show that the effects of wall can be observed as soon as $\xi \lesssim 0.5$ since a deviation is noted from the expected linear profile (dotted line). Our simulations accurately match those from Ganatos et al. (1982) even in the very near-wall region. Wall effects grow as the particle comes closer to the wall and particle velocity rapidly departs from the linear profile and asymptotes to the wall velocity $U_{w}$.

Wall interactions also result in a significant increase in the particle stresslet as seen in Fig. 1(b) which presents the non-dimensional hydrodynamic stresslet $S_{x y} / S_{x y, \infty}$ where $S_{x y, \infty}$ is the stresslet of a unique particle in an unbounded domain $S_{x y, \infty}=10 / 3 \pi \eta a^{3} \dot{\gamma}$. Similarly to particle velocity, particle stresslet rapidly increases in the near-wall region. Predictions are in good agreement with theoretical works by Sangani et al. (2011) (solid line in Fig. 1(b)). This curve is given by the following asymptotic development, valid for $\xi<0.15$

$$
\frac{S_{x y}}{S_{x y, \infty}}=\frac{0.847 \ln \xi^{-1}-0.41+1.44 \xi \ln \xi^{-1}-0.3 \xi}{0.2 \ln \xi^{-1}+0.6376}
$$

This relation suggests that the stresslet $S_{x y}$ remains finite at contact and can reach $0.847 / 0.2 \approx 4.2$. This means that the stresslet $S_{x y}$ of a particle in contact with a wall is 4.2 times larger than the stresslet this particle would have in an unbounded domain.

\section{Effect of confinement : results and discussion}

The objective of this study is to investigate the role of walls on suspensions, especially on rheology. Numerical simulations of suspensions are performed for different volume fractions in the range $0.1 \leq \phi \leq 0.55$. The computational domain is a cell of size $L_{x}, L_{y}$, $L_{z}$ in the direction of velocity, velocity gradient and vorticity, respectively. The channel width $L_{y}$ will be varied whereas $L_{x}=20 a$ and $L_{z}=20 a$. For $L_{y}=20 a$ (the most investigated case) and $\phi=0.5$, the total number of particles is about 1,000 . We recall that the magnitude of confinement is described by parameter $\kappa$ which is the ratio between channel width $L_{y}$ and particle radius $\kappa=L_{y} / a$. An unbounded suspension has $\kappa \rightarrow \infty$ whereas the minimum value $\kappa=2$ is reached for a gap having the same size as the particle. A shear flow of magnitude $\dot{\gamma}$ is imposed by moving upper and lower walls with opposite velocities. 
Periodic boundary conditions are used in $x$ (velocity direction) and $z$ (vorticity direction). The numerical parameters are a grid spacing $\Delta=a / 5$ and a time step $5 \cdot 10^{-4} \dot{\gamma}^{-1}$. All runs are started using random hard-sphere equilibrium configurations obtained from a Monte-Carlo procedure. For steady results, the initial strain $(\dot{\gamma} t<50)$ is discarded and the computation is continued for another 100 150 $\dot{\gamma} t$. Rheological properties are based upon the computation of the Batchelor particle stress $\Sigma_{i j}^{p}$ (Batchelor \& Green 1972) which is the contribution of particles to the bulk suspension stress. It is further decomposed as

$$
\Sigma_{i j}^{p}=\Sigma_{i j}^{h}+\Sigma_{i j}^{c}
$$

with

$$
\begin{aligned}
& \Sigma_{i j}^{h}=n\left\langle S_{i j}^{h}\right\rangle \\
& \Sigma_{i j}^{c}=n\left\langle S_{i j}^{c}\right\rangle
\end{aligned}
$$

where $S_{i j}^{h}$ and $S_{i j}^{c}$ are the hydrodynamic and contact stresslets, respectively, $n$ is the number density of particles and brackets $\langle\cdot\rangle$ indicate an ensemble average. For a linear shear flow, the relative viscosity $\eta_{r}=\eta_{s} / \eta$ (where $\eta_{s}$ is the suspension viscosity) reads

$$
\eta_{r}=1+\frac{\Sigma_{x y}^{p}}{\eta \dot{\gamma}}
$$

The normal stress differences are given by

$$
\begin{aligned}
& N_{1}=\Sigma_{x x}^{p}-\Sigma_{y y}^{p} \\
& N_{2}=\Sigma_{y y}^{p}-\Sigma_{z z}^{p}
\end{aligned}
$$

\subsection{Wall-induced structuring}

The onset of specific structures in the suspension can be monitored using orientational order metrics such as $Q_{6}$ and $C_{6}$. The metrics $Q_{6}$ is based on the spherical harmonics $Y_{l m}(\theta, \varphi)$ of the orientational bond angles $(\theta, \varphi)$ between particles and is defined as (Rintoul \& Torquato 1996)

$$
Q_{6}=\sqrt{\frac{4 \pi}{13} \sum_{m=-6}^{6}{\overline{Y_{6 m}}}^{2}}
$$

where $\overline{Y_{6 m}}$ represents the average $Y_{6 m}(\theta, \varphi)$ over the neighbouring particles. For a completely disordered system, $Q_{6}=0$ whereas the maximal value $Q_{6}^{F C C} \approx 0.575$ is reached for a face-centred cubic structure. Another metrics $C_{6}$ has been introduced by Kulkarni \& Morris (2009) to specifically track hexagonal structures. It is based on the threedimensional pair-correlation function $g(r, \theta, \varphi)$ and given as

$$
C_{6}=\max _{\psi} \frac{\int_{0}^{2 \pi} g(2 a, \pi / 2, \varphi) \cos [6(\varphi-\psi)] d \varphi}{\int_{0}^{2 \pi} g(2 a, \pi / 2, \varphi) d \varphi}
$$

The angle $\psi$ accounts for a possible tilt of the structure around the $x$-axis. In our computations however, we have always found $\psi=0$ meaning that the obtained hexagonal structure is untilted. $C_{6}$ is 0 for a disorder random system and reaches 1 for a perfect hexagonal lattice. Note that since particles are rough, they experience actual contact and in Eq. (3.8), the pair-correlation function is therefore computed for $r \leq 2 a+h_{r}$.

Figure 2 plots the evolution of $C_{6}$ and $Q_{6}$ with respect to the suspension volume fraction $\phi_{b u l k}$. Computations are performed for frictionless particles $\left(\mu_{d}=0\right)$ and confinement $\kappa=20$. Both parameters have a similar profile with very small values in dilute regimes 


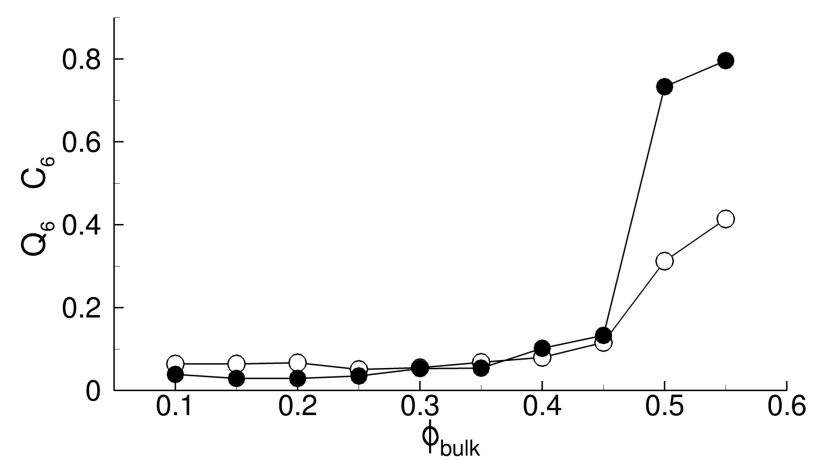

FiguRE 2. Order parameters $Q_{6}(\circ)$ and $C_{6}(\bullet)$ as a function of volume fraction $\left(\kappa=20 ; \mu_{d}=0\right)$.

(a)

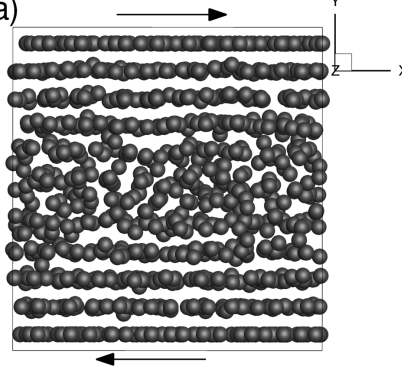

(b)

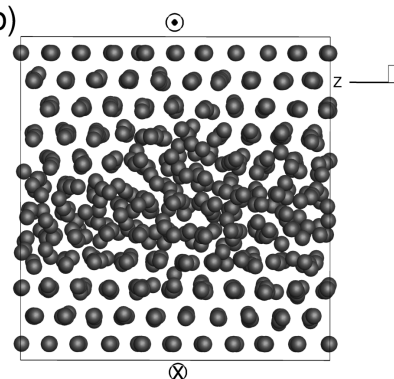

Figure 3. Particle snapshots $\left(\phi_{b u l k}=0.5 ; \kappa=20 ; \mu_{d}=0\right):(a)$ side view ; (b) end view. For visualization, particle radius is half the actual size.

and an abrupt increase for a volume fraction in the range $0.45 \sim 0.5$. For this confinement $\kappa=20$, this marks the transition between disordered and ordered states. High $C_{6}$ values show that the system preferentially crystallizes into an hexagonal structure, in accordance with previous studies (Yeo \& Maxey 2010c; Kulkarni \& Morris 2009). Friction has a weak effect on this structuring as will be seen later.

This structuring is easily noticed on particle snapshots as illustrated in Fig. 3. This figure plots an instantaneous particle configuration at volume fraction $\phi_{\text {bulk }}=0.5$ and $\kappa=20$ in the shear plane $x-y$ (side view, (a)) and in the velocity gradient-vorticity plane $y-z$ (end view, (b)). For the sake of clarity, particles are represented at half their actual size. Particles are seen to form layers in the vicinity of the walls whereas the core of the suspension remains disordered. The end view (plane $y-z$ ) in Fig. 3(b) clearly shows an hexagonal structure close to walls with a given particle being at the centre of an hexagon formed by six neighbours.

\subsection{Wall effects on volume fraction}

Since we are concerned with wall-bounded flows with periodicity imposed in $x$ and $z$ directions, average quantities depend on vertical position $y$. As proposed by Yeo \& Maxey $(2010 b)$, an average volume fraction $\langle\phi(y)\rangle$ can be defined as

$$
\langle\phi(y)\rangle=\frac{1}{L_{x} L_{z}}\left\langle\iint \chi(x, y, z) \mathrm{d} x \mathrm{~d} z\right\rangle
$$

where $\chi$ is the particle indicator function which is 1 in the particle and 0 elsewhere. Note that $\langle\phi(y)\rangle$ is rather an areal fraction but it is known from stereology theory to be equal to the volume fraction (Delesse principle). Figure 4 presents this local volume 


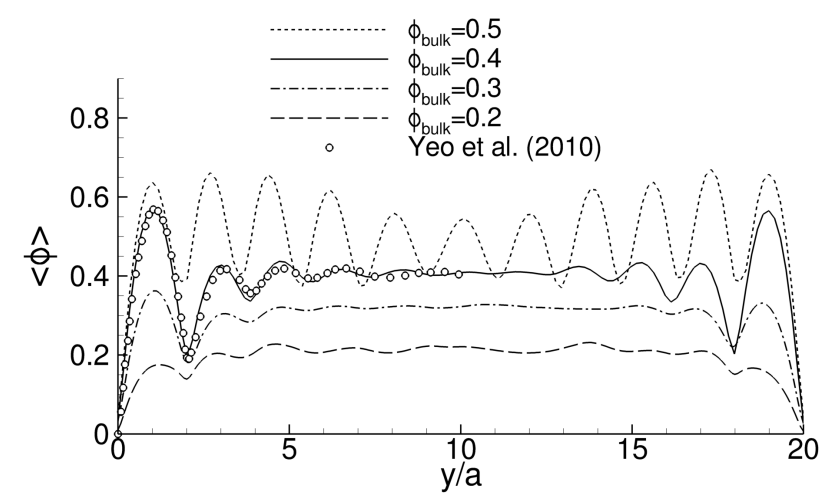

FiguRE 4. Local volume fraction $\langle\phi(y)\rangle$ in the channel width for $\kappa=20$ and bulk fractions $\phi_{\text {bulk }}=(0.2,0.3,0.4,0.5)$. Open symbols are computations by Yeo \& Maxey $(2010 b)$.

fraction $\langle\phi(y)\rangle$ for four different bulk fractions $\phi_{b u l k}$ in the case $\kappa=20$ and frictionless particles. Some simulation results by Yeo \& Maxey $(2010 b)$ at $\phi_{b u l k}=0.4\left(\mu_{d}=0\right)$ are also plotted. Local peaks in the wall region indicate the presence of a stable particle layering, which is also attested in other computations (Yeo \& Maxey 2010b; Kromkamp et al. 2006) or experiments (Blanc et al. 2013; Cheng et al. 2011; Snook et al. 2015; Pieper \& Schmid 2016). This layering exists irrespective of the bulk volume fraction $\phi_{b u l k}$. However, for moderate fractions such as $\phi_{b u l k}=0.2$, it is much less pronounced and is noted only for the first two layers $(y / a<4)$. In present case $\kappa=20$, and for $\phi_{\text {bulk }}$ below 0.5 , there is still a flat profile in the core. In this core region, the suspension is devoid of wall effects and is therefore expected to behave like an unbounded suspension. On the opposite, for $\phi_{b u l k}=0.5$, wall effects are dominant across the whole channel. This value is consistent with the rapid increase in order parameters as seen in Fig. 2. The size of the wall-structured region $e_{\text {wall }}$ can be estimated from the spatial variations of $\langle\phi(y)\rangle$. A rough criterion used here is to define $e_{\text {wall }}$ such that $a|d\langle\phi(y)\rangle / d y| / \phi_{\text {bulk }}>0.1$. To address dense suspensions, it is necessary to consider large domains so as to allow this wall structuring to freely develop. Results are compiled in Fig. 5 for frictionless particles with the chosen confinement $\kappa$ specified for each volume fraction. For $\phi_{b u l k}<0.4$, the size of the wall structuring grows mildly with $\phi_{b u l k}$, from $3 a$ to $6 a$. It however increases abruptly for $\phi_{\text {bulk }} \gtrsim 0.4$, even though larger domains are considered. For $\phi_{b u l k} \geq 0.52$, there is a marked organized structure across the whole domain despite the wide channel investigated $L_{y}=80 a$. In that case, $e_{\text {wall }}$ is set to $L_{y} / 2=40 a$. This is not the physical value but it only indicates that the size could not be determined since the whole suspension is ordered. Simulations in larger domains were not performed. This result is reminiscent of simulations from Kulkarni \& Morris (2009) and Sierou \& Brady (2002) who showed that even in unbounded suspensions, there is a crystallization of the system for $\phi_{b u l k}$ between 0.5 and 0.55 (although for $\phi \geq 0.6$, the system could become disordered again; we have yet not considered such high fractions). This behaviour is close to a system of hard-spheres with a freezing point at $\phi_{f} \approx 0.49$. Since it can occur in infinite domains, the complete ordering noted in our simulations at $\phi_{\text {bulk }}=0.52$ and $\phi_{\text {bulk }}=0.55$ in large domains may not be due solely to walls. Monte-Carlo simulations have showed that the crystallisation of hard sphere systems is faster when walls are present (Volkov et al. 2002). It can therefore be considered as a wall-induced crystallisation since it is promoted by an existing local ordering. This crystallisation is not experimentally attested in suspensions which might be due to the present use of monodisperse particles while experiments always consider slightly polydisperse particles. 


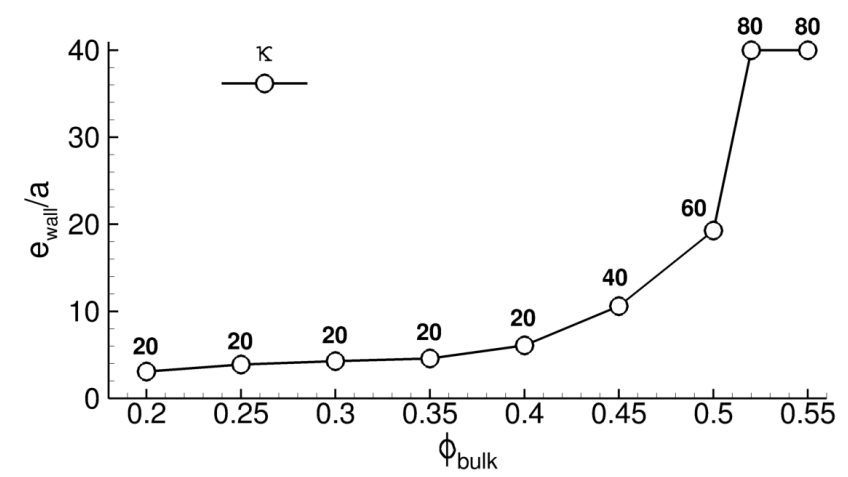

FiguRE 5. Size of the wall-structured region $e_{\text {wall }}$ as a function of bulk volume fraction $\phi_{\text {bulk }}$ $\left(\mu_{d}=0\right)$. The confinement $\kappa$ considered is provided in bold number in the figure. For the last two points at $e_{\text {wall }} / a=40$, the whole suspension is ordered.

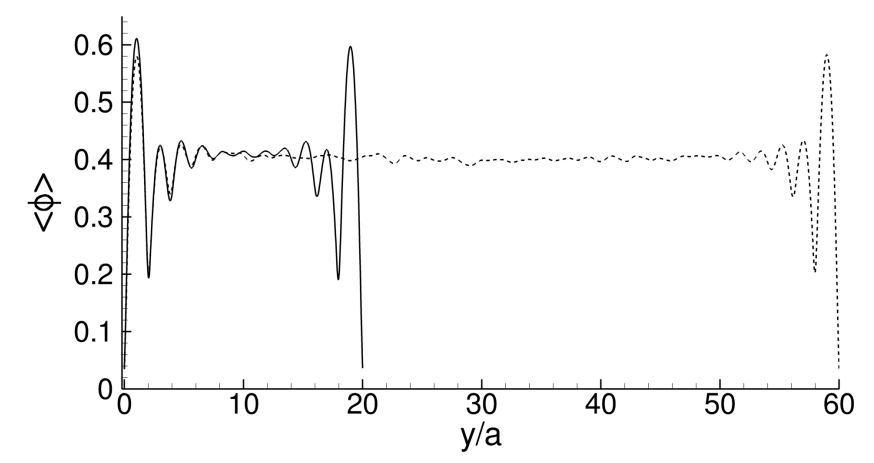

Figure 6. Volume fraction profile $\langle\phi(y)\rangle$ for $\kappa=20$ (solid lines) and $\kappa=60$ (dotted lines). Simulations at $\phi_{b u l k}=0.4$ and $\mu_{d}=0$.

When the wall-induced ordering can develop freely, it does not seem to depend much on the channel height. Simulations at $\phi_{b u l k}=0.4$ were conducted in a moderately confined suspension $(\kappa=20)$ and a weakly confined suspension $(\kappa=60)$. Results are presented in Fig. 6 and show very similar structure near walls (peak heights and positions). This absence of domain size influence was also reported by other simulations (Yeo \& Maxey $2010 b$ ) and experiments (Eral et al. 2009).

Let us conclude this section on volume fraction by investigating the role of friction. The same suspension at $\kappa=20$ and $\phi_{\text {bulk }}=0.4$ is computed for frictionless $\left(\mu_{d}=0\right)$ and frictional $\left(\mu_{d}=0.5\right)$ particles. The obtained volume fraction profiles are provided in Fig. 7 and globally share similar characteristics. However, frictional particles result in less marked peaks in the wall region indicating a slightly weakened structuring. This is much clearer on the first particle layer where the local volume fraction is lower in case of friction. Intuitively, tangential contact forces between particles promote a more active momentum exchange between adjacent layers, which may contribute to destabilizing well-ordered layers. This may also be in connection with a higher diffusion noted for frictional particles (Gallier 2014). 


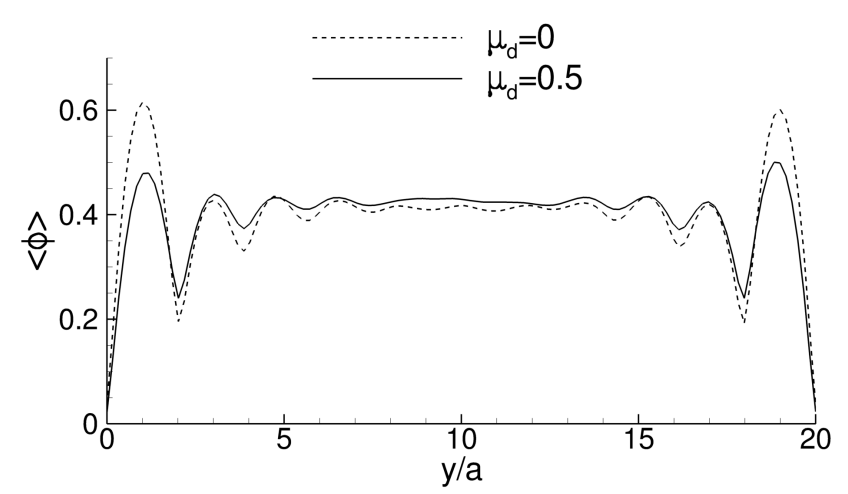

FiguRE 7. Volume fraction profile $\langle\phi(y)\rangle$ for $\mu_{d}=0$ (dotted lines) and $\mu_{d}=0.5$ (solid lines). Simulations at $\phi_{\text {bulk }}=0.4$ and $\kappa=20$.

\subsection{Wall effects on particle velocity}

The effects of walls on axial particle velocity $U$ can similarly be investigated using the following particle-phase average velocity $\langle U(y)\rangle$ (Yeo \& Maxey 2010b)

$$
\langle U(y)\rangle=\frac{\left\langle\iint \chi(x, y, z) U^{(k)} \mathrm{d} x \mathrm{~d} z\right\rangle}{\left\langle\iint \chi(x, y, z) \mathrm{d} x \mathrm{~d} z\right\rangle}
$$

with $U^{(k)}$ the translational velocity at the centre of particle $k$. Figure 8 presents this velocity profile, normalized by the wall velocity $U_{w}$, for a suspension at $\kappa=20$ and $\phi_{b u l k}=0.4$ for frictionless $\left(\mu_{d}=0\right)$ and frictional particles $\left(\mu_{d}=0.5\right)$. Both profiles are similar with a linear evolution in the core of the suspension and a strong effect of walls on the velocity. The role of friction is limited and tends to smooth velocity variations, in accordance with the previous effects on volume fraction. Because of significant wall-induced layering, the velocity is quasi-constant within a layer and this forms plateaus in the velocity profile close to the walls. This is particularly noted for the first layer and it is then progressively damped farther in the flow until the expected linear profile is found in the centre of the suspension. In the first layer $(0<y<2 a)$, the particle velocity is close to the wall velocity $U_{w}$ because of lubrication forces. It is yet not exactly equal to wall velocity mostly because of roughness. Since lubrication tangential forces scale as $\log \xi$, they are bounded by $\log \xi_{r}$ where $\xi_{r}=h_{r} / a$ is the non-dimensional roughness.

In their work, Jana et al. (1995) studied wall slip in suspensions and proposed an experimental correlation for the slip velocity $u_{e}$ as

$$
u_{e}=\frac{\eta_{r}}{8} \dot{\gamma} a
$$

where $\dot{\gamma}$ represents here the local shear rate and $\eta_{r}$ is the overall suspension viscosity. This wall slip leads to a shear rate that is smaller than the macroscopic prescribed shear rate $\dot{\gamma}_{b u l k}=2 U_{w} / L_{y}$. This shear rate $\dot{\gamma}_{J a n a}$ reads $2\left(U_{w}-u_{e}\right) / L_{y}$ and, accounting for Eq. (3.11), can be expressed as $\dot{\gamma}_{J a n a}=\dot{\gamma}_{b u l k}-\dot{\gamma}_{J a n a} \eta_{r} / 4 \kappa$. This eventually gives $\dot{\gamma}_{J a n a}=\dot{\gamma}_{b u l k} /(1+$ $\left.\eta_{r} / 4 \kappa\right) \approx \dot{\gamma}_{\text {bulk }}\left(1-\eta_{r} / 4 \kappa\right)$. From the viscosity $\eta_{r}$ taken from our simulations at $\phi_{\text {bulk }}=0.4$ and $\kappa=20$, Jana's model gives $\dot{\gamma}_{\text {Jana }} / \dot{\gamma}_{\text {bulk }} \approx 0.93$ in the frictionless case $\left(\eta_{r}=6.0\right)$ and 0.89 in the frictional case $\left(\eta_{r}=9.1\right)$. These values can be compared to the numerical shear rate obtained by a linear regression of $\langle U(y)\rangle$ in the suspension homogeneous core $\dot{\gamma}_{\text {core }}=(d\langle U(y)\rangle / d y)_{5 \leq y / a \leq 15}$. From the profiles of Fig. 8, this regression yields $\dot{\gamma}_{\text {core }} / \dot{\gamma}_{\text {bulk }} \approx 0.96$ in the frictionless case and $\dot{\gamma}_{\text {core }} / \dot{\gamma}_{\text {bulk }} \approx 0.89$ in the frictional case, in good agreement with $\dot{\gamma}_{\text {Jana }}$ from Jana's model. 


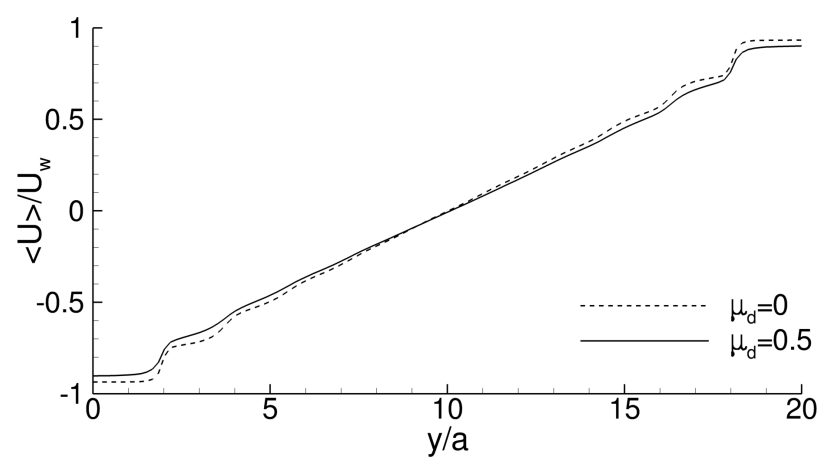

Figure 8. Particle-phase axial velocity $\langle U(y)\rangle / U_{w}$ for frictionless particles $\mu_{d}=0$ (dotted lines) and frictional particles $\mu_{d}=0.5$ (solid lines). Simulations at $\phi_{b u l k}=0.4$ and $\kappa=20$.

Close to the walls, we have mentioned that the particle velocity is not exactly equal to the wall velocity $U_{w}$. The scaled slip velocity $\left|-U_{w}-\left\langle U_{y=0}\right\rangle\right| / \dot{\gamma} a$ at the lower wall $y=0$ is about 0.6 and 1.0 in the frictionless and frictional case, respectively. It is not zero - meaning that particles are not stuck on walls - nor 0.5 which would be the expected velocity of a particle rolling without slip on the wall (assuming $\Omega_{z}=-\dot{\gamma} / 2$ ). This occurs mostly because lubrication tangential force is bounded by $\log \xi_{r}$. This can be checked by investigating the case where particle roughness is discarded for interactions with the walls (however, roughness is still kept for particle-particle interactions). Particles can thus come arbitrarily close to the walls with the possibility of vanishing distance between particles and walls. As seen in Fig. 9 (frictionless case), the velocity of the first particle layer is now much closer to the wall velocity. The slip velocity $\left|-U_{w}-\left\langle U_{y=0}\right\rangle\right| / \dot{\gamma} a$ is reduced irrespective of friction and is about 0.3 (frictionless particles) and 0.4 (frictional particles) which is lower than in the case of wall-particle roughness (0.6 and 1.0 in the frictionless and frictional case, respectively). Because wall-particle gap $\xi$ is reduced, lubrication tangential forces are higher and increase particle entrainment by the moving walls. In the case $\xi=0$ (actual contact), theoretical studies by Chaoui \& Feuillebois (2003) show that particles would be indeed stuck to the wall with translational velocity $U=U_{w}$ and zero rotational velocity. A linear regression of particle velocity in the core region gives that $\dot{\gamma}_{\text {core }} / \dot{\gamma}_{\text {bulk }} \approx 1$ in the frictionless case and 0.96 in the frictional one, suggesting a reduction of apparent wall slip.

Finally, we investigate the effects of walls on particle rotation rate. Figure 10 presents the particle-phase angular velocity $\left\langle\Omega_{z}(y)\right\rangle$ scaled by $\dot{\gamma}_{b u l k}$ for frictionless and frictional particles. In the suspension homogeneous core, a value close to the expected $-\dot{\gamma}_{\text {bulk }} / 2$ is found. The noted effect of friction can be explained by the different core shear rate because of wall slip. Indeed, scaling by the core shear rate $\dot{\gamma}_{\text {core }}$ leads to the same value $\left\langle\Omega_{z}(y)\right\rangle / \dot{\gamma}_{\text {core }} \approx-0.54$, irrespective of friction. Note that unlike volume fraction, the profile shows no plateau even in the suspension core, suggesting that the domain might be too small here to obtain a local homogeneity of the rotational velocity. A plateau is indeed found but for larger channel height. Walls are found to play a significant role because tangential lubrication interactions hinder particle rotation. In the frictionless case, the rotation rate is roughly divided by two compared to the suspension core. In the theoretical case of a single smooth sphere at non-dimensional distance $\xi=\xi_{r}=5.10^{-3}$, the expected rotation rate (scaled by shear rate) is 0.249 (Chaoui \& Feuillebois 2003) which is close to the average rotation rate in the first layer, about 0.275 . In the frictional case, the additional tangential contact force imposes a torque on particles and increases rotation. 


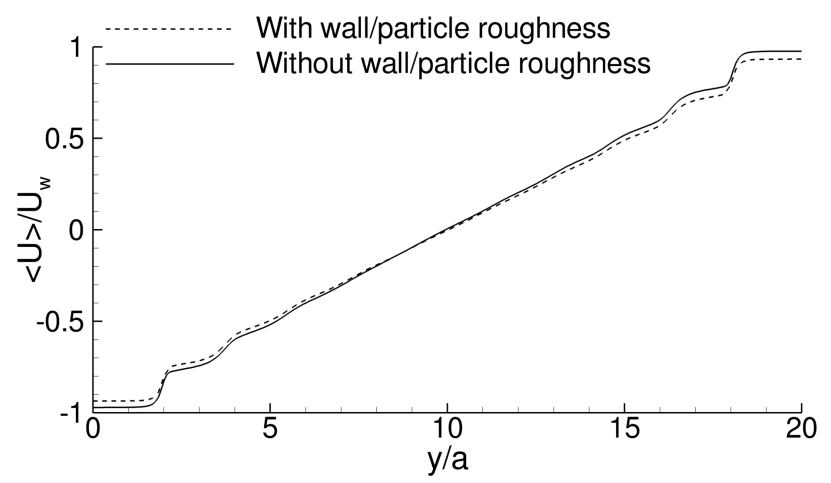

FiguRE 9. Particle-phase axial velocity $\langle U(y)\rangle / U_{w}$ with and without wall-particle roughness. Simulations at $\phi_{\text {bulk }}=0.4, \kappa=20$ and $\mu_{d}=0$.

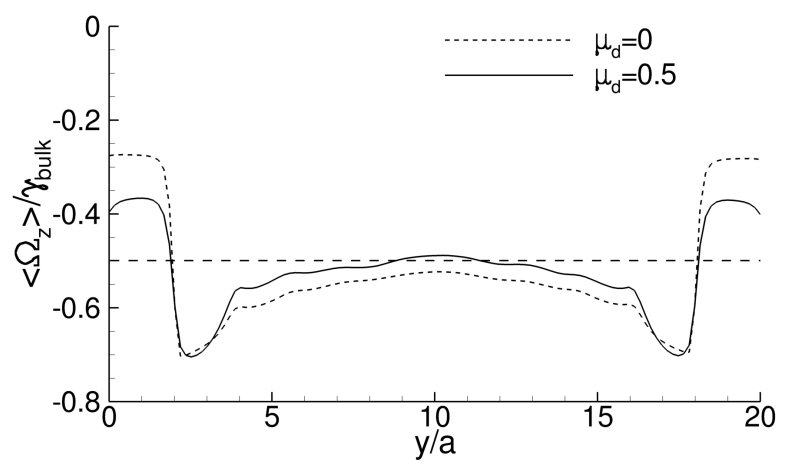

FIGURE 10. Particle-phase angular velocity $\left\langle\Omega_{z}(y)\right\rangle / \dot{\gamma}_{b u l k}$ for frictionless particles $\mu_{d}=0$ (dotted lines) and frictional particles $\mu_{d}=0.5$ (solid lines). Simulations at $\phi_{b u l k}=0.4$ and $\kappa=20$. Dashed line is $-\dot{\gamma}_{\text {bulk }} / 2$.

On the walls, particles roll with partial slip since the ratio $\left|a\left\langle\Omega_{z}\right\rangle /\left(\left\langle U_{y=0}\right\rangle+U_{w}\right)\right|$ is less than 1 . Interestingly, this ratio is about 0.4 for both frictionless and frictional particles. This value is consistent with the theoretical case of a single smooth sphere at wall distance $\xi_{r}$ where this ratio is about 0.52 (Chaoui \& Feuillebois 2003). Maximal rotation rate is reached between first and second layer $(y \approx 2.5 a)$ with $\left\langle\Omega_{z}\right\rangle \approx-0.7 \dot{\gamma}_{\text {bulk }}$.

\subsection{Wall effects on viscosity}

Wall-induced structuring involves some suspension thixotropy with significant transients until a steady regime is reached. This unsteady behaviour is noticed during strains about 10 or more. Figure 11 simultaneously plots the evolution of the relative viscosity $\eta_{r}$ and the order parameter $Q_{6}$ with strain for a suspension at $\phi_{\text {bulk }}=0.5$ and $\kappa=20$. The initial configuration (at $\gamma=0$ ) is a random hard-sphere configuration in equilibrium. At the very beginning, the viscosity suddenly increases from $\eta_{r} \approx 7$ (the viscosity of a random configuration) to about 13 before decreasing to a steady value. The evolution of $Q_{6}$ mirrors the viscosity which hints at a strong link between viscosity and suspension ordering. The average steady-state is reached after a strain of about 40 . This is close to the value of approximately 30 found in a similar simulation by Yeo \& Maxey $(2010 c)$. This characteristic strain is however significantly larger than a typical strain of $\approx 1$ needed for the defor- 

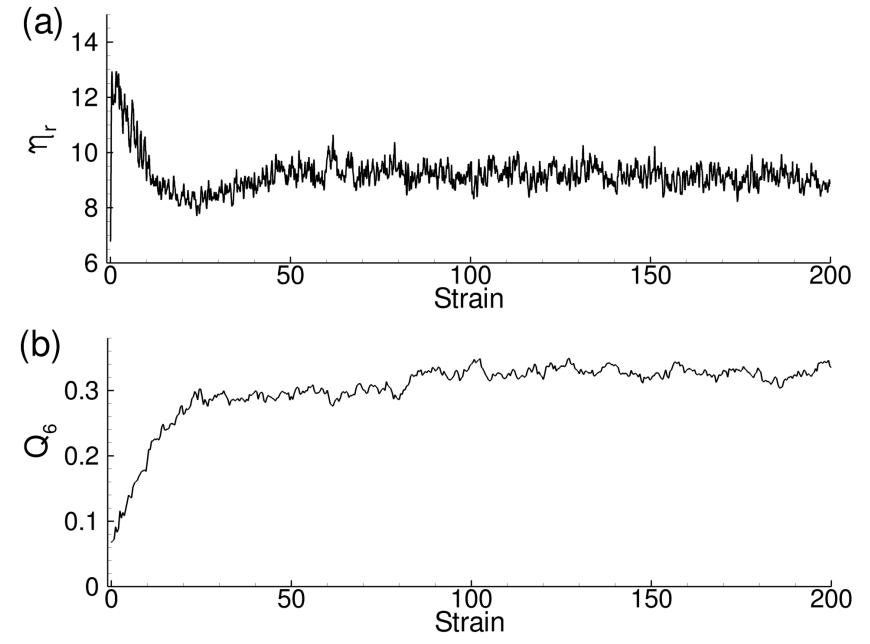

FIGURE 11. Relative viscosity $\eta_{r}$ (a) and parameter $Q_{6}$ (b) as a function of strain $\gamma=\dot{\gamma}_{b u l k} t$. Simulations at $\phi_{b u l k}=0.5, \kappa=20$ and $\mu_{d}=0$.

mation of the suspension microstructure due to the imposed shear. The latter basically corresponds to the rapid initial transient between the starting isotropic microstructure and a deformed anisotropic microstructure.

When a steady regime is eventually reached, confinement alters the suspension viscosity depending on the bulk volume fraction $\phi_{b u l k}$. Results presented in Fig. 12 show the suspension relative viscosity $\eta_{r}$ for $\kappa=20$ and two friction coefficients $\mu_{d}=0$ and $\mu_{d}=0.5$. As noted previously for this confinement $\kappa=20$, the wall structuring spreads across the whole suspension as soon as $\phi_{\text {bulk }}=0.45 \sim 0.5$. In Fig. 12, this basically corresponds to the point where the viscosity curve $\eta_{r}\left(\phi_{\text {bulk }}\right)$ shows an inflexion point. For very concentrated suspension $\left(\phi_{b u l k}=0.55\right)$, the suspension viscosity decreases irrespective of friction. This decrease in the viscosity is also reported in other simulations (Yeo \& Maxey 2010c; Kulkarni \& Morris 2009) and is a consequence of particle layering. For the highest volume fractions - where the suspension is strongly ordered - results are expected to depend much on confinement as well as how channel size and particle size commensurate (Yeo \& Maxey 2010c; Bian et al. 2014). Such commensurability effects will be discussed in Sec. 3.7 .

The spatial evolution of stresses in the suspension is studied using a particle-phase average stress defined as

$$
\left\langle\Sigma_{i j}(y)\right\rangle=n \frac{\left\langle\iint \chi(x, y, z) S_{i j}^{(k)} \mathrm{d} x \mathrm{~d} z\right\rangle}{\left\langle\iint \chi(x, y, z) \mathrm{d} x \mathrm{~d} z\right\rangle}
$$

where $S_{i j}^{(k)}$ is the stresslet (hydrodynamic, contact, or the sum thereof) of particle $k$ and $n$ the number density of particles in the whole domain. Note that this is not a real local stress but rather a local stresslet having the dimension of a stress due to the particle density $n$ pre-factor. Figure 13 presents the local particle tangential stress $\left\langle\Sigma_{x y}^{p}(y)\right\rangle$ (referred to as total stress in the figure legend) as well as the hydrodynamic tangential stress $\left\langle\Sigma_{x y}^{h}(y)\right\rangle$ and contact tangential stress $\left\langle\Sigma_{x y}^{c}(y)\right\rangle$. Stresses are scaled by the fluid stress $\eta \dot{\gamma}$ and are here computed for a suspension at $\phi_{b u l k}=0.4$ and $\kappa=20$ for frictionless (a) and frictional particles (b). We recall that the total particle stress $\Sigma_{x y}^{p}$ is split up into an hydrodynamic stress $\Sigma_{x y}^{h}$ and a contact stress $\Sigma_{x y}^{c}$ by virtue of Eq. (3.1). Let us first 


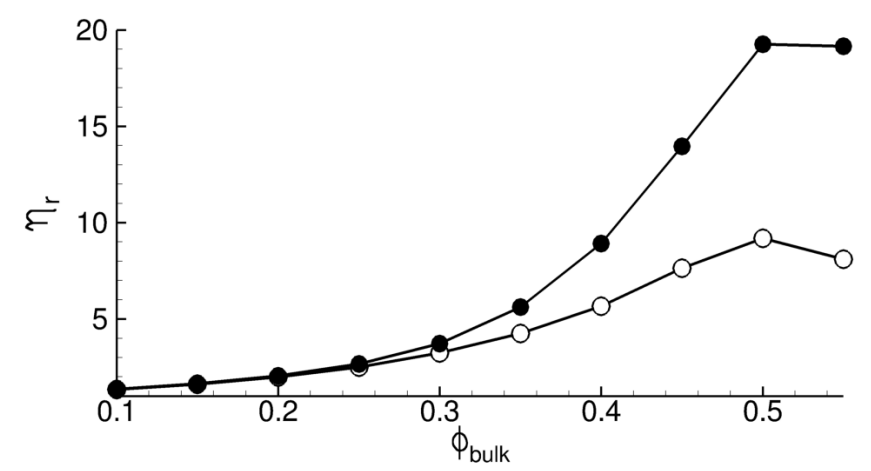

Figure 12. Relative viscosity $\eta_{r}$ as a function of volume fraction $(\kappa=20)$ for friction coefficients $\mu_{d}=0(\circ)$ and $\mu_{d}=0.5(\bullet)$.

consider the frictionless case (Fig. 13(a)). As expected, stresses are relatively constant in the homogeneous core. The maximum in the particle stress $\Sigma_{x y}^{p}$ (therefore, $\eta_{r}$ ) is reached between the first and second layer, at $y \approx 2.5 a$. This results from a large contribution from both hydrodynamics and contact. In the first layer $(0 \leq y \leq 2 a)$, the hydrodynamic contribution remains important due to wall-particle lubrication interactions. The contact contribution is here negligible since contact forces are mostly in the normal direction $y$ in the frictionless case, which results in a very small $\Sigma_{x y}^{c}$. However, for $2 a \leq y \leq 3 a$, this corresponds to some particles located somewhere between the first two layers. Such particles are expected to experience stronger contacts, which explains the increase in the contact stress. Friction (Fig. 13(b)) does not profoundly modify those conclusions. Stress levels in the suspension core are larger mostly because of contacts as already detailed in Gallier et al. (2014b). The stress peak between first and second layer is less visible than in the frictionless case due to a higher level of hydrodynamic and contact stress at the wall. Because of frictional contact, the tangential contact force does involve an additional contact contribution on the $x y$ stress. It is important to recall that the chosen average Eq. (3.12) accounts for an average stress density since the local stresslet is scaled by the local volume fraction (denominator in Eq. (3.12)). Because of the wall depleted zone, the local volume fraction is small in the near-wall region so that the contribution of this wall region to the overall suspension stress is weak.

By and large, the profiles of $\left\langle\Sigma_{x y}^{p}(y)\right\rangle$ are moderately affected by walls. Therefore, the viscosity $\eta_{r}^{\kappa}$ of a wall-bounded suspension (at confinement $\kappa$ ) may not be that different from the viscosity $\eta_{r}^{\infty}$ expected for an unbounded homogeneous suspension. This unbounded viscosity $\eta_{r}^{\infty}$ is here computed in the homogeneous core of a suspension in a large domain. Note that $\eta_{r}^{\kappa}$ is calculated based on the prescribed macroscopic shear rate $\dot{\gamma}_{\text {bulk }}$ whereas $\eta_{r}^{\infty}$ is computed using the local shear rate $\dot{\gamma}_{\text {core }}$ in the core of the suspension. This viscosity ratio is plotted in Fig. 14 for $\kappa=20$ as a function of volume fraction for frictionless $\left(\mu_{d}=0\right)$ and frictional $\left(\mu_{d}=0.5\right)$ particles. This ratio is always close to 1 , regardless of the friction coefficient, except for $\phi_{b u l k}=0.5$ where a strong layering results in a viscosity decrease. Note that this viscosity ratio is slightly below 1 for dilute suspensions. Actually, the tangential stress ratio is indeed about 1 , so that $\eta_{r}^{\kappa} / \eta_{r}^{\infty} \approx \dot{\gamma}_{\text {core }} / \dot{\gamma}_{\text {bulk }}$. Because of wall slip, this shear rate ratio $\dot{\gamma}_{\text {core }} / \dot{\gamma}_{\text {bulk }}$ is lower than 1 and is about 0.98 for low volume fractions, which explains the values of viscosity ratio in Fig. 14. 
(a)

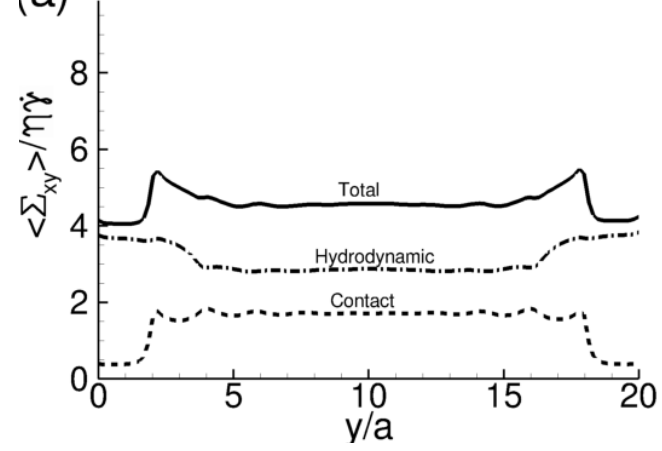

(b)

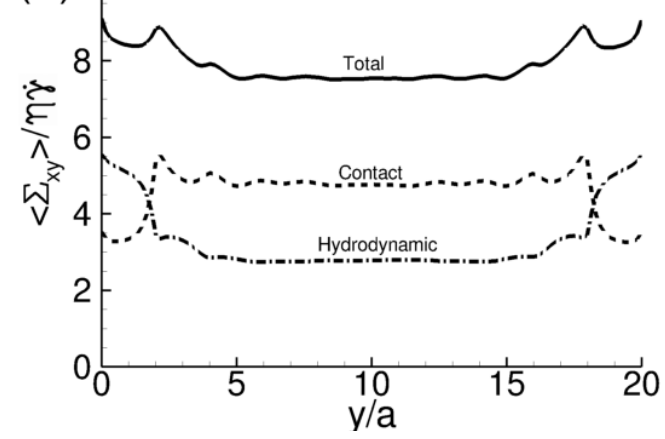

FIGURE 13. Particle-phase particle stress $\left\langle\Sigma_{x y}^{p}(y)\right\rangle / \eta \dot{\gamma}$ (solid lines), hydrodynamic stress $\left\langle\Sigma_{x y}^{h}(y)\right\rangle / \eta \dot{\gamma}$ (dash-dotted lines) and contact stress $\left\langle\Sigma_{x y}^{c}(y)\right\rangle / \eta \dot{\gamma}$ (dotted lines) in a suspension at $\phi_{\text {bulk }}=0.4$ and $\kappa=20$ for two friction coefficients : $\mu_{d}=0$ (a) ; $\mu_{d}=0.5$ (b)

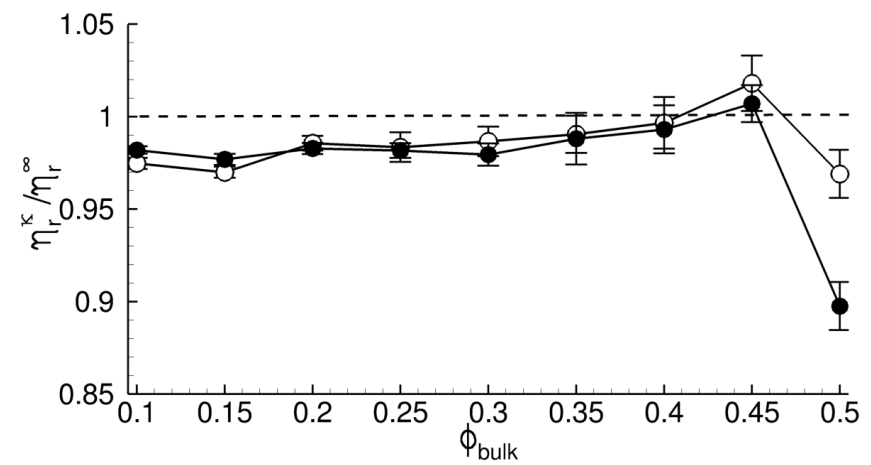

FiguRE 14. Ratio of bounded to unbounded viscosity $\eta_{r}^{\kappa} / \eta_{r}^{\infty}$ as a function of volume fraction for $\mu_{d}=0(\circ)$ and $\mu_{d}=0.5(\bullet)$ for $\kappa=20$.

\subsection{Wall effects on normal stress differences}

A similar analysis is conducted for the normal stress differences $N_{1}$ and $N_{2}$ due to their importance in rheology. The local profiles of the particle-phase average $\left\langle N_{1}(y)\right\rangle$ and its contact contribution $\left\langle N_{1}^{c}(y)\right\rangle$ (scaled by the fluid stress $\eta \dot{\gamma}$ ) are presented in Fig. 15 for a frictionless (a) and frictional case (b) for a suspension at $\phi_{\text {bulk }}=0.4$ confined at $\kappa=20$. Unlike viscosity, a strong effect of walls is observed which can locally modify the sign of $\left\langle N_{1}\right\rangle$. In the homogeneous core, $\left\langle N_{1}\right\rangle / \eta \dot{\gamma}$ is about -1 but close to the walls, it can increase to +1 or even +4 in case of friction. Irrespective of friction, the contact contribution remains very small in the core $\left(\left\langle N_{1}^{c}\right\rangle \ll\left\langle N_{1}\right\rangle\right)$ but this is no longer the case in the vicinity of the walls where it represents the major contribution, i.e. $\left\langle N_{1}\right\rangle \approx\left\langle N_{1}^{c}\right\rangle$. Furthermore, near the walls, $\left\langle N_{1}^{c}\right\rangle$ changes its sign from negative to positive while the hydrodynamic part $\left\langle N_{1}^{h}\right\rangle$ becomes close to zero.

In order to gain further insight on this strong wall effect, Fig. 16 plots the contact stresses $\left\langle\Sigma_{x x}^{c}(y)\right\rangle$ and $\left\langle\Sigma_{y y}^{c}(y)\right\rangle$ for this case. Those results show that $\left\langle\Sigma_{x x}^{c}\right\rangle \approx\left\langle\Sigma_{y y}^{c}\right\rangle$ in the suspension core from which $\left\langle N_{1}^{c}\right\rangle \approx 0$ is expected. This has been shown to arise from a uniform distribution of contacts in the compression region (Gallier et al. 2014b). Conversely, simulations predict a substantial decrease of $\left|\left\langle\Sigma_{x x}^{c}\right\rangle\right|$ close to the walls whereas $\left|\left\langle\Sigma_{y y}^{c}\right\rangle\right|$ remains similar (frictionless case) or even slightly increases (frictional case). Contact forces in the $y$ direction are only weakly affected and walls mostly act to reduce 

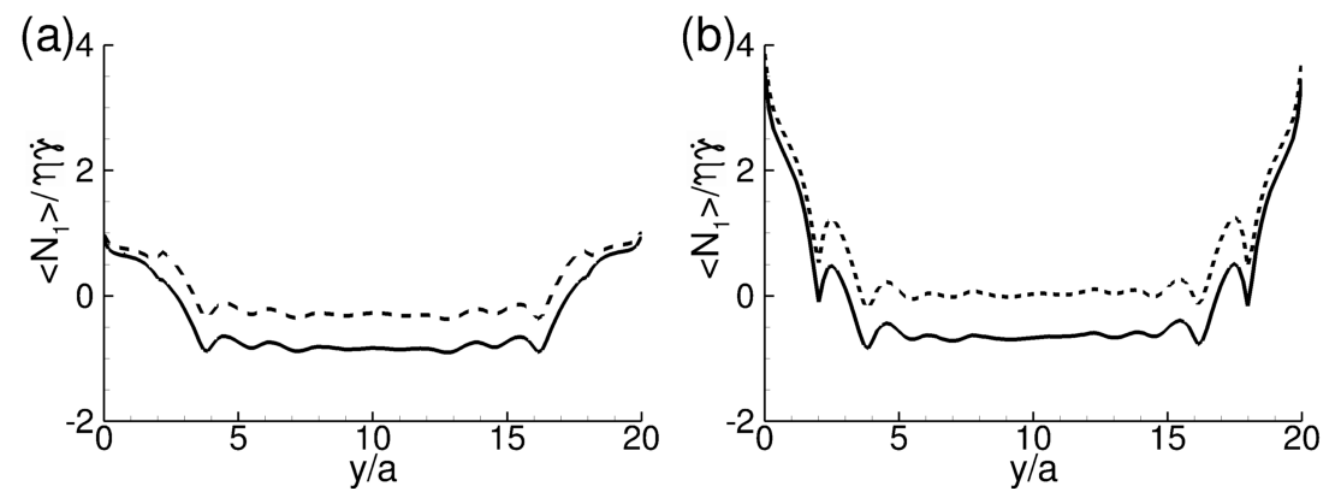

Figure 15. Particle-phase average $\left\langle N_{1}(y)\right\rangle / \eta \dot{\gamma}$ (solid lines) and $\left\langle N_{1}^{c}(y)\right\rangle / \eta \dot{\gamma}$ (dotted lines) in a suspension at $\phi_{b u l k}=0.4$ and $\kappa=20$ for two friction coefficients : $\mu_{d}=0$ (a); $\mu_{d}=0.5$ (b).
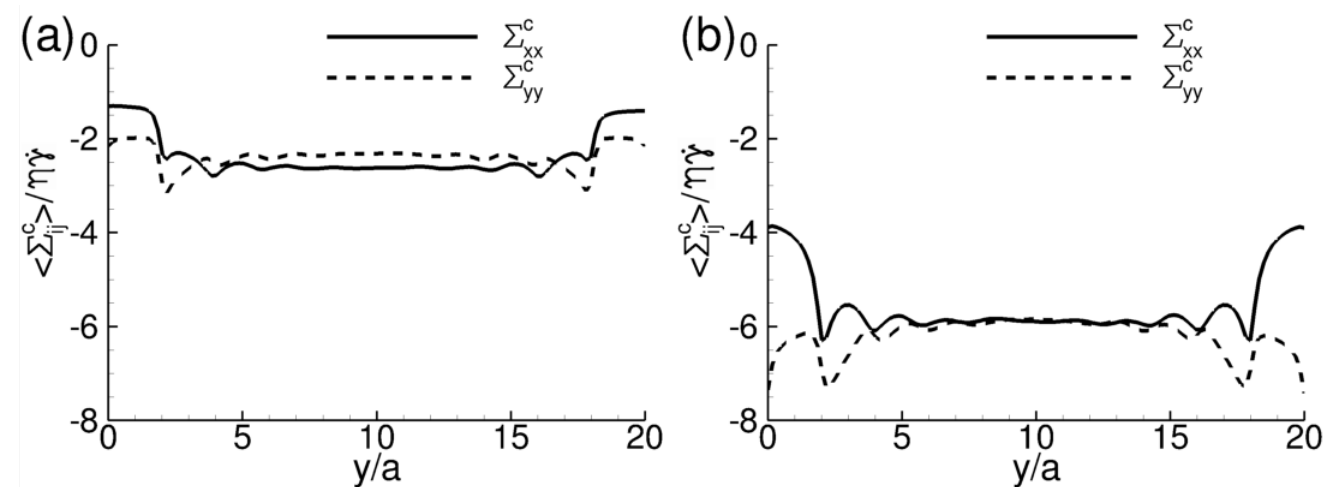

FIGURE 16. Particle-phase average contact stresses $\left\langle\Sigma_{x x}^{c}(y)\right\rangle / \eta \dot{\gamma}$ (solid lines) and $\left\langle\Sigma_{y y}^{c}(y)\right\rangle / \eta \dot{\gamma}$ (dotted lines) in a suspension at $\phi_{b u l k}=0.4$ and $\kappa=20$ for two friction coefficients : $\mu_{d}=0$ (a) ; $\mu_{d}=0.5$ (b).

contact forces in the velocity direction $x$. This is related to a layered configuration : particles in the first layer have similar velocities so that particles hardly interact in this direction. A decrease in $\left|\left\langle\Sigma_{x x}^{c}\right\rangle\right|$ is therefore expected. This induces high positive values of $\left\langle N_{1}^{c}\right\rangle$ which, in turn, involve the positive $\left\langle N_{1}\right\rangle$ observed in Fig. 15.

From the marked effect of walls on $N_{1}$, we can expect the $N_{1}^{\kappa}$ obtained in a bounded suspension to be significantly different from its unbounded homogeneous counterpart $N_{1}^{\infty}$. The ratio $N_{1}^{\kappa} / N_{1}^{\infty}$ is presented in Fig. 17 for a suspension at $\kappa=20$. This ratio is always smaller than 1 meaning that $N_{1}$ in a bounded suspension is always lower than in an infinite suspension. This ratio decreases with friction as well as with volume fraction, mostly for $\phi>0.2$. An important result is that this ratio is negative for dense suspensions, meaning that the overall suspension $N_{1}$ becomes positive due to confinement. It is therefore possible that the combined action of walls and friction is liable to explain some experimental results showing almost zero or positive values of $N_{1}$ (Couturier et al. 2011; Dbouk et al. 2013; Gamonpilas et al. 2016). This point will be reconsidered hereinafter.

We now move to the second normal stress difference $N_{2}$. The local $N_{2}$ profile in the suspension is presented similarly in Fig. 18 with both total and contact contributions. The contact $N_{2}^{c}$ (dotted lines in Fig. 18) can be hardly distinguished from the total $N_{2}$ meaning that $N_{2}$ is entirely due to contacts $\left(N_{2} \approx N_{2}^{c}\right)$ and that the hydrodynamic contribution is negligible $\left(N_{2}^{h} \approx 0\right)$. Note that this stands for the whole suspension (Gallier 


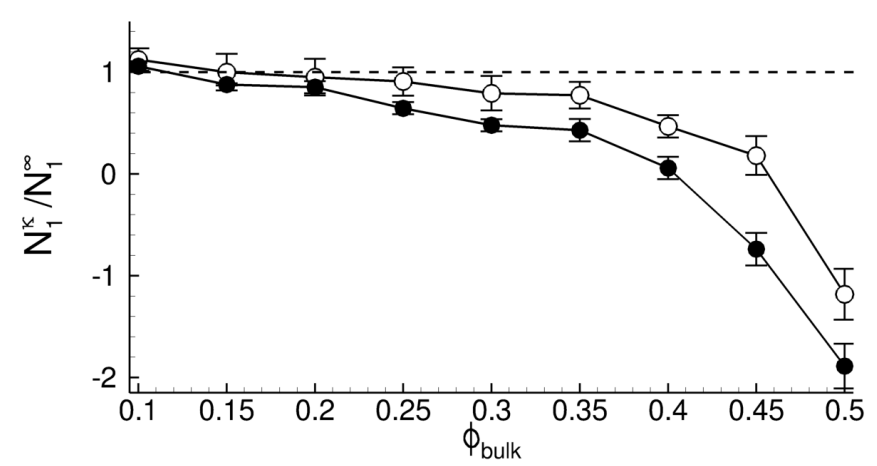

FiguRE 17. Ratio of bounded to unbounded $N_{1}^{\kappa} / N_{1}^{\infty}$ as a function of volume fraction for $\mu_{d}=0(\circ)$ and $\mu_{d}=0.5(\bullet)$ for $\kappa=20$.
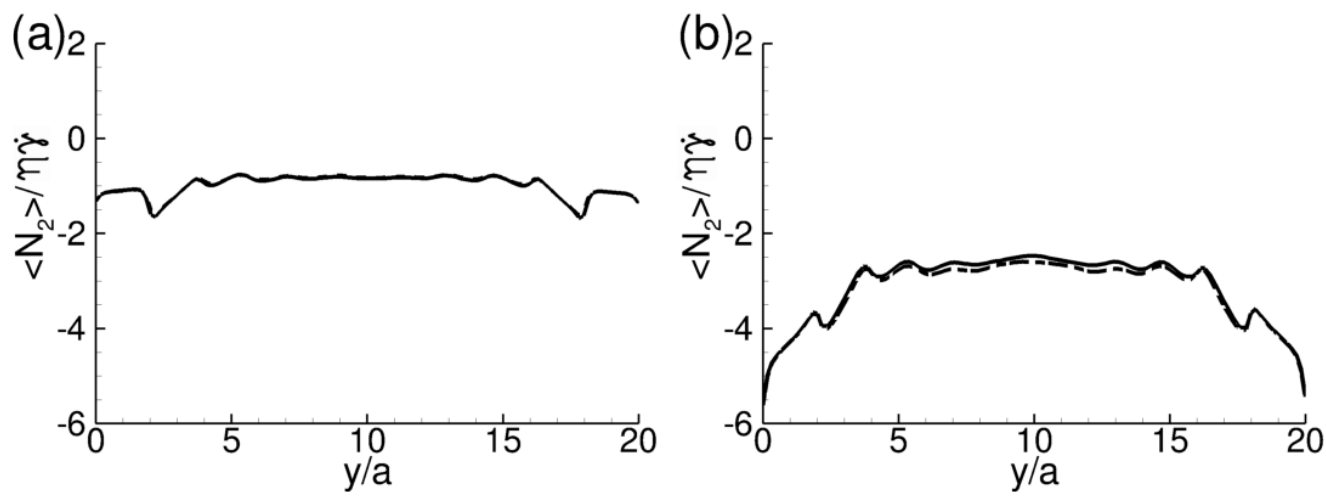

Figure 18. Particle-phase average $\left\langle N_{2}(y)\right\rangle / \eta \dot{\gamma}$ (solid lines) and $\left\langle N_{2}^{c}(y)\right\rangle / \eta \dot{\gamma}$ (dotted lines) in a suspension at $\phi_{b u l k}=0.4$ and $\kappa=20$ for two friction coefficients : $\mu_{d}=0$ (a); $\mu_{d}=0.5$ (b).

et al. 2014b) but also locally everywhere in the flow. For frictionless particles (Fig. 18a), $N_{2}$ is almost constant across the suspension whereas in the frictional case (Fig. 18b), a significant increase in $\left|N_{2}\right|$ is noticed close to the walls. This is due to the particle structuring in the vorticity direction (see Fig. 3) which decreases contact forces in this direction and $\left|\Sigma_{z z}^{c}\right|$ accordingly.

Those results show that $N_{2}$ is moderately affected by walls unlike $N_{1}$. This is especially true in the frictionless case. For frictional particles, the variation of $N_{2} / \eta \dot{\gamma}$ in the wall region is significant $(\approx-3)$ and is almost of the same order of magnitude as for $N_{1}(\approx+5)$. But because $N_{1}$ is small, its relative variation is much higher than for $N_{2}$. Furthermore, there is no change in the sign of $N_{2}$ as opposed to $N_{1}$. Consequently, the overall $N_{2}^{\kappa}$ computed in a confined suspension might not significantly differ from its unbounded counterpart $N_{2}^{\infty}$. This is confirmed in Fig. 19 for a suspension confined at $\kappa=20$. The ratio $N_{2}^{\kappa} / N_{2}^{\infty}$ is about 1 for dilute and moderately dense suspensions. For frictionless particles and dilute suspensions (say, $\phi_{\text {bulk }} \leq 0.2$ ), it can however be lower than 1 but this possibly comes from large statistical errors - as shown by the error bars - primarily because values of $N_{2}$ are extremely small in that case. This statistical error is here computed as the standard deviation over statistically independent intervals of 20 30 strain units each. For volume fractions above 0.4 , the $N_{2}$ for a confined suspension can be greater by up to $50 \%$ compared to an homogeneous suspension and with a 


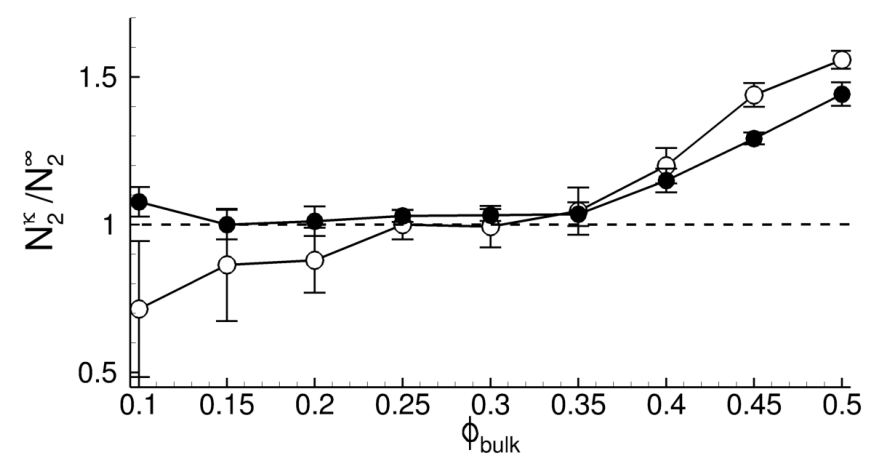

FiguRE 19. Ratio of bounded to unbounded $N_{2}^{\kappa} / N_{2}^{\infty}$ as a function of volume fraction for $\mu_{d}=0(\circ)$ and $\mu_{d}=0.5(\bullet)$ for $\kappa=20$.

weak effect of friction.

\subsection{Normal stress differences : comparison with experiments}

One of the motivations of this work was to investigate to which extent the confinement of a suspension can explain discrepancies between experiments, especially on $N_{1}$. A first study - described in a previous work (Gallier et al. 2014b) - has shown that friction leads to a decrease in $\left|N_{1}\right|$ and can help match available experiments and simulations. However, it was concluded that friction itself can not result in positive $N_{1}$ as measured in some experiments (Dbouk et al. 2013). The present results moreover suggest a significant role of walls - even in moderately confined suspensions $(\kappa=20)$ - leading to positive values of $N_{1}$. Figure 20 compiles experimental results on normal stress differences (normalized by shear stress $\left.\tau=\eta_{r} \eta \dot{\gamma}\right)$ compared to our frictional $\left(\mu_{d}=0.5\right)$ simulations in a bounded $(\kappa=20)$ and unbounded suspension. The experiments are taken from six sources from the literature (Zarraga et al. 2000; Singh \& Nott 2000; Dbouk et al. 2013; Dai et al. 2013; Couturier et al. 2011; Gamonpilas et al. 2016) using different techniques. These seem to be the major experiments measuring simultaneously $N_{1}$ and $N_{2}$ (other experiments are available but for $N_{2}$ only, e.g. Garland et al. (2013)). Let us begin with $N_{2}$ (Figure 20b) since it deserves less attention. As expected, the effect of confinement is limited and does not significantly change from unbounded suspension. In any case, simulation results are close to experiments, which are moreover relatively consistent between them. Concerning $N_{1}$ (Figure 20a), simulations show a change of sign for dense suspensions, typically for $\phi \gtrsim 0.4$, and $N_{1}$ becomes largely positive above. Confinement can be considered as a potential source for experimental discrepancy since experiments are conducted in different geometries and confinements. It is however still difficult to predict the experimental data from Dbouk et al. (2013) that are visible in Fig. 20(a) as the most positive values ( $\diamond$ symbols). Even at $\phi \approx 0.3 \sim 0.4$, the measured $N_{1} / \tau$ is larger than 0.1 whereas simulations predict $N_{1} / \tau \approx 0$ for this range of volume fractions. Note that these latter experiments are performed at $\kappa \approx 27$, which is not far from our simulations at $\kappa=20$. A puzzling point is that their study proposed additional experiments in much less confined suspensions $(\kappa \approx 100)$ but found similar $N_{1}$ results. This suggests that measurements are weakly dependent on confinement unlike our simulations since for $\kappa \approx 100$, we expect results close to our $\kappa=\infty$ results (see (o) symbols in Fig. 20). A possible explanation is that these experiments - which rely on a measurement of $\Sigma_{y y}$ on the rheometer walls describe a flow configuration which is different from the overall suspension (in the sense 

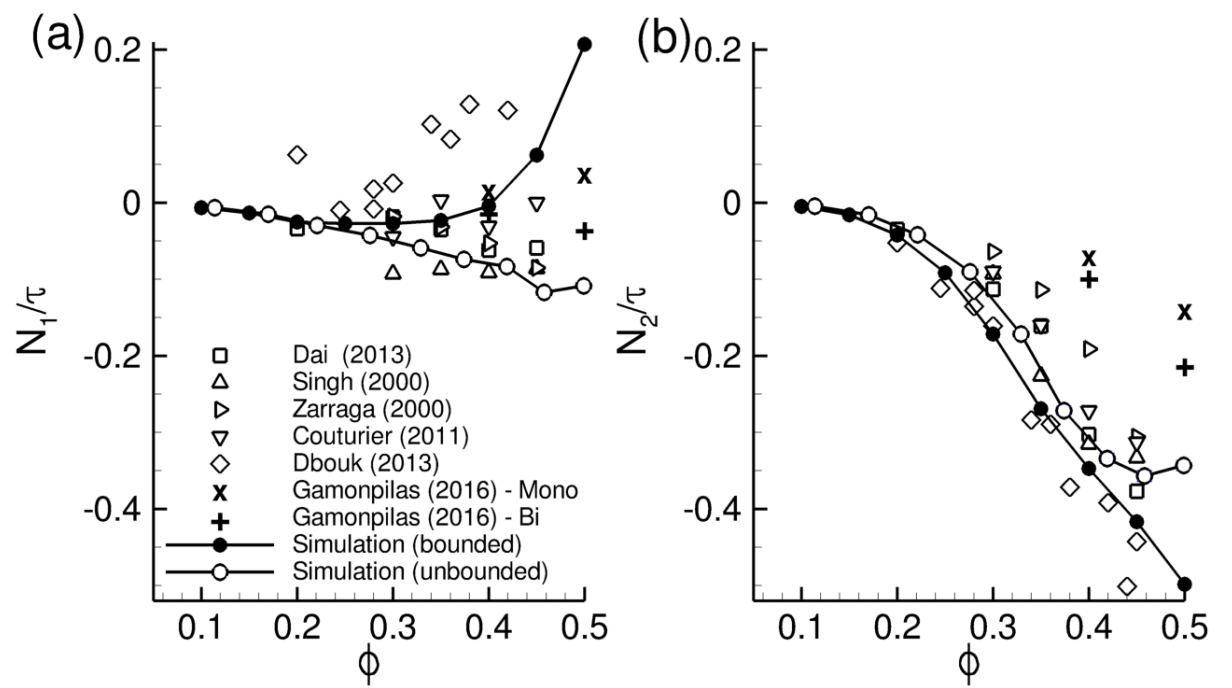

FiguRE 20. Normal stress differences $N_{1}$ (a) and $N_{2}$ (b) normalized by shear stress $\tau$ as a function of volume fraction for a frictional suspension $\mu_{d}=0.5$ : bounded suspension $\kappa=20(\bullet)$ and unbounded suspension (o). Experiments (symbols) compile results from Zarraga et al. (2000); Singh \& Nott (2000); Dbouk et al. (2013); Dai et al. (2013); Couturier et al. (2011); Gamonpilas et al. (2016). Data from Gamonpilas et al. (2016) are for monomodal particles (Mono) and bimodal mixture $(\mathrm{Bi})$.

of a volume average over the whole domain) but is rather characteristic of the near-wall ordered state. Since this local structuring is independent of confinement (see Fig. 6), this could explain why experiments from Dbouk et al. (2013) are unaffected by confinement. The question of which stress is measured in experiments seems still open and should deserve attention in future works. As a final remark, it is interesting to note that results by Gamonpilas et al. (2016) show a difference in the sign of $N_{1}$ depending whether the suspension is monodisperse (positive $N_{1}$ ) or bidisperse (negative $N_{1}$ ). Monodispersity is well known to promote ordering - as seen in present simulations - which is consistent with our conclusion that $N_{1}$ is positive because of wall-induced layering.

\subsection{Effect of confinement on rheology}

Most of the above analysis is conducted with a moderate confinement $\kappa=20$ and we here intend to evaluate the effect of confinement $\kappa$ on rheology, namely viscosity and normal stress differences. The forthcoming computations are run only in the frictionless case $\left(\mu_{d}=0\right)$ and for a single volume fraction $\phi_{b u l k}=0.4$. As noted previously, the friction enhances wall effects but does not seem to modify the underlying physics, which explains that only frictionless particles are addressed here.

Figure 21 presents the effect of the confinement $\kappa$ on the computed viscosity $\eta_{r}$ in the range $6 \leq \kappa \leq 60$. Two different regimes are noted with a transition at $\kappa_{c}$ about $12 \sim 15$ : an oscillating regime for $\kappa \lesssim \kappa_{c}$ and a monotonic regime for $\kappa \gtrsim \kappa_{c}$. The value of $\kappa_{c}$ is expected to correspond to a wall-induced ordering that spans across the whole channel. For $\phi_{b u l k}=0.4$, the size of the wall-structured region $e_{\text {wall }}$ was found to be about $6 a$ (see Fig. 5). This would give $\kappa_{c} \approx 12$, which is consistent with the present results.

In the very confined regime $\left(\kappa \lesssim \kappa_{c}\right)$, the viscosity exhibits an oscillating behaviour (see also the inset in Fig. 21). This is related to commensurability effects as already noted by Yeo \& Maxey $(2010 c)$. Their simulations show that order parameter $C_{6}$ and particle 


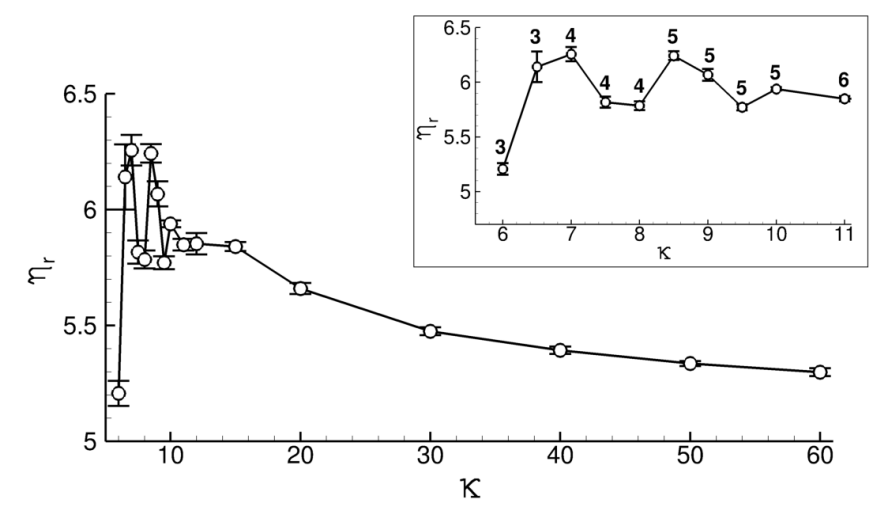

FiguRE 21. Effect of confinement $\kappa$ on viscosity $\eta_{r}$ at $\phi_{b u l k}=0.4$ and $\mu_{d}=0$ in the range $6 \leq \kappa \leq 60$. (Inset : range $6 \leq \kappa \leq 11$; the bold numbers indicate the number of layers).

pressure $\Pi$ are very sensitive to the commensurability of the ordered structures with the channel height. Unsurprisingly, our results reveal a similar behaviour on viscosity which fluctuates depending on how the structure is frustrated by the narrow channel. The bold numbers in the inset indicate the number of particle layers across the channel height. The maximum in the viscosity seems to correspond to a situation when the number of layers has just increased by one. In that case, the distance between layers is smaller making particles less mobile and the suspension more ordered.

For the monotonic regime $\left(\kappa \gtrsim \kappa_{c}\right)$, the viscosity slightly decreases monotonically towards an asymptotic value which is obtained for $\kappa \gtrsim 50 \sim 60$. This is consistent with experiments by Zarraga et al. (2000) who have found that the asymptotic value is reached for $\kappa \gtrsim 40$. The viscosity computed at $\kappa=30$ is only off by $3 \%$ compared to $\kappa=60$, suggesting that a confinement ratio $\kappa$ larger than 30 is suitable for reliable viscosity measurements. The fact that viscosity decreases when $\kappa$ increases is also found in simulations (Bian et al. 2014; Davit \& Peyla 2008), as well as in the experiments from Peyla \& Verdier (2011) and the theoretical expressions by Sangani et al. (2011). On the contrary, simulations by Yeo \& Maxey (2010b) and experiments by Zarraga et al. (2000) show an increase in viscosity with increasing channel height. There seems to be a predominant role of volume fraction since the studies showing a viscosity decrease are for more dilute suspensions (range $0.05 \sim 0.4$ ) whereas the viscosity increase is noticed in denser regimes (range $0.4 \sim 0.45$ ). A definite conclusion would require simulations for a wide range of volume fractions, which were not done here. But it seems that the effects are quite complex and possibly result from a subtle interplay between wall-enhanced hydrodynamic interactions, wall-induced structuring, and apparent wall slip.

Figure 22 similarly presents the effect of the confinement $\kappa$ on the normal stress differences $N_{1}$ and $N_{2}$ scaled by the fluid stress $\eta \dot{\gamma}$. Analogously to viscosity, two regimes are clearly noticed. For $\kappa \gtrsim \kappa_{c}, N_{1} / \eta \dot{\gamma}$ and $N_{2} / \eta \dot{\gamma}$ show a monotonic evolution towards an asymptotic value. The confined regime $\kappa \lesssim \kappa_{c}$ displays a non-monotonic behaviour in connection with commensurability effects. The fluctuations are large and $N_{1}$ can be strongly positive while $N_{2}$ always remains negative. It is interesting to note that $N_{1}$ and $N_{2}$ have mirrored behaviours : an increase in $N_{1}$ is always related to a decrease in $N_{2}$. This is expected from our previous conclusions about the effect of layering on normal stresses : particle layers result in an increase in $\Sigma_{x x}^{p}$ and $\Sigma_{z z}^{p}$ and a relatively unchanged $\Sigma_{y y}^{p}$. As a consequence, $N_{1}=\Sigma_{x x}^{p}-\Sigma_{y y}^{p}$ is expected to increase while $N_{2}=\Sigma_{y y}^{p}-\Sigma_{z z}^{p}$ decreases concurrently. 


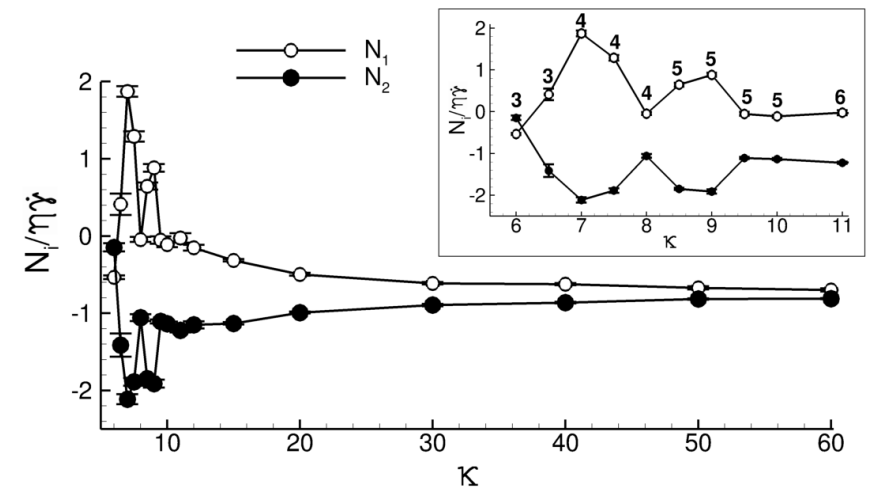

Figure 22. Effect of confinement $\kappa$ on normal stress differences $N_{1} / \eta \dot{\gamma}(\mathrm{o})$ and $N_{2} / \eta \dot{\gamma}(\bullet)$ at $\phi_{\text {bulk }}=0.4$ and $\mu_{d}=0$ in the range $6 \leq \kappa \leq 60$. (Inset : range $6 \leq \kappa \leq 11$; the bold numbers indicate the number of layers).

\section{Conclusions}

In this paper, we have presented three-dimensional numerical simulations of concentrated suspensions in a wall-bounded shear flow. Simulations rely on a fictitious domain method including long-range hydrodynamics, particle-particle and wall-particle lubrication forces, and contact frictional forces. Notably, wall-lubrication corrections are proposed on rheological quantities, which does not seem to be reported in previous similar computations.

Walls lead to a local hexagonal structuring of particles. The size of this layered zone depends on volume fraction and is only weakly affected by friction. For a confinement $\kappa=20$ (ratio between channel width and particle radius), this region represents half of the suspension volume at $\phi_{\text {bulk }}=0.4$ but the whole domain as soon as $\phi_{\text {bulk }} \gtrsim 0.5$. For $\phi_{\text {bulk }} \gtrsim 0.52$, the system completely crystallizes even in very large domains $\left(L_{y}=80 a\right)$. This result is expected for monodisperse particles and the effect of polydispersity was not investigated. The wall structuring is relatively slow to develop with characteristic strains of $\mathcal{O}(10)$. It involves wall slip, leading to a reduced shear rate in the suspension core which seems consistent with the empirical model of Jana et al. (1995). For perfectly smooth wall, the wall slip is mostly due to particle roughness that limits the wall-particle gap and lubrication intensity accordingly.

Wall-induced ordering is shown to have a limited impact on viscosity and second normal stress difference $N_{2}$ at least for moderately confined suspensions $(\kappa=20)$. Conversely, it significantly affects the first normal stress difference $N_{1}$. Friction enhances this effect which is shown to be due to a large decrease in the contact normal stress $\left|\Sigma_{x x}^{c}\right|$ because of particle layering in the wall region. Our simulations suggest that confinement and friction can promote positive values of $N_{1}$. The obtained results seem in better agreement with recent $N_{1}$ measurements (Couturier et al. 2011; Dbouk et al. 2013) and eventually highlight the importance of friction and confinement for quantitative predictions of actual suspensions.

Some future work will have to address size polydispersity since most experimental suspensions have finite polydispersity. This is needed to extend the relevance of our results beyond single-sized spheres, especially regarding experiments. Polydispersity is likely to reduce ordering and may alter the balance between confinement effects per se and confinement effects through wall-induced layering. This could also help understand 
results by Gamonpilas et al. (2016) who found different normal stress differences between monodisperse and bidisperse systems.

\section{Acknowledgements}

This work has been funded by the French Defence Procurement Agency (DGA).

\section{Appendix A. Wall resistance functions}

Asymptotic expressions for particle-wall resistance functions $X^{A}, Y^{A}, Y^{B}, X^{C}, Y^{C}$, $Y^{G}$ and $Y^{H}$ can be found in various sources of the literature and have been recently compiled by Yeo \& Maxey $(2010 b)$. Note that in their paper, there seems to be a typographical error for the $\mathcal{O}(1)$ term in $Y^{H}$ which is 0.0916 instead of 0.923 (see Bossis et al. (1991)).

$$
\begin{aligned}
\frac{X^{A}}{6 \pi a} & =\xi^{-1}+\frac{1}{5} \ln \xi^{-1}+\frac{1}{21} \xi \ln \xi^{-1}+0.8193 \\
\frac{Y^{A}}{6 \pi a} & =\frac{8}{15} \ln \xi^{-1}+\frac{64}{375} \xi \ln \xi^{-1}+0.9557 \\
\frac{Y^{B}}{4 \pi a^{2}} & =-\frac{3}{15} \ln \xi^{-1}-\frac{43}{125} \xi \ln \xi^{-1}+0.3852 \\
\frac{X^{C}}{8 \pi a^{3}} & =-\frac{1}{2} \xi \ln \xi^{-1}+1.2021 \\
\frac{Y^{C}}{8 \pi a^{3}} & =\frac{2}{5} \ln \xi^{-1}+\frac{66}{125} \xi \ln \xi^{-1}+0.3720 \\
\frac{Y^{G}}{4 \pi a^{2}} & =\frac{7}{10} \ln \xi^{-1}+\frac{221}{250} \xi \ln \xi^{-1}-0.9230 \\
\frac{Y^{H}}{8 \pi a^{3}} & =-\frac{1}{10} \ln \xi^{-1}+\frac{2}{250} \xi \ln \xi^{-1}+0.0916
\end{aligned}
$$

where $\xi$ is the gap between particle surface and wall normalized by particle radius $a$. In order to obtain the missing functions $X^{G}, X^{P}$ and $Y^{M}$, we start from their general expressions for two particles having different size (Kim \& Karrila 1991; Jeffrey 1992) and we note $\beta$ the size ratio. A variable change is first needed because in the theoretical two-sphere expressions, distance $\xi$ is non-dimensional using the average radius $a(1+\beta) / 2$ while we want to keep $a$ for a wall-particle interaction. Then, the limit $\beta \rightarrow \infty$ is taken in the obtained expression. The $\mathcal{O}(1)$ non-singular term is taken from Jeffrey (1992) for $\beta=100$, which is the highest value available. The final asymptotic expressions are

$$
\begin{aligned}
\frac{X^{G}}{4 \pi a^{2}} & =\frac{3}{2} \xi^{-1}-\frac{6}{5} \ln \xi^{-1}+0.268 \\
\frac{X^{P}}{4 \pi a^{2}} & =\frac{3}{2} \xi^{-1}-\frac{6}{5} \ln \xi^{-1}-0.552 \\
\frac{Y^{M}}{\frac{20}{3} \pi a^{3}} & =\frac{24}{25} \ln \xi^{-1}+\frac{1182}{625} \xi \ln \xi^{-1}-0.685
\end{aligned}
$$

Note that there seem to be no data available in the intermediate distance regime so that those asymptotic expressions are always used. 


\section{REFERENCES}

Batchelor, G.K. \& Green, J.T. 1972 The hydrodynamic interaction of two small freelymoving spheres in a linear flow field. Journal of Fluid Mechanics 56 (02), 375-400.

Bian, X., Litvinov, S., Ellero, M. \& Wagner, N.J. 2014 Hydrodynamic shear thickening of particulate suspension under confinement. Journal of Non-Newtonian Fluid Mechanics 213, 39-49.

Blanc, F., Lemaire, E., Meunier, A. \& Peters, F. 2013 Microstructure in sheared nonbrownian concentrated suspensions. Journal of Rheology 57 (1), 273-292.

Blanc, F., Peters, F. \& Lemaire, E. 2011 Experimental signature of the pair trajectories of rough spheres in the shear-induced microstructure in noncolloidal suspensions. Physical review letters 107 (20), 208302.

Bossis, G.., Meunier, A \& Sherwood, J.D. 1991 Stokesian dynamics simulations of particle trajectories near a plane. Physics of Fluids A: Fluid Dynamics (1989-1993) 3 (8), 18531858.

Brady, J.F. \& Bossis, G. 1988 Stokesian dynamics. Annual Review of Fluid Mechanics 20 (1), $111-157$.

Chaoui, M. \& Feuillebois, F. 2003 Creeping flow around a sphere in a shear flow close to a wall. The Quarterly Journal of Mechanics and Applied Mathematics 56 (3), 381-410.

Cheng, X., McCoy, J.H., Israelachvili, Jacob N. \& Cohen, I. 2011 Imaging the microscopic structure of shear thinning and thickening colloidal suspensions. Science 333 (6047), 1276-1279.

Coussot, P. 2005 Rheometry of pastes, suspensions, and granular materials: applications in industry and environment. John Wiley \& Sons.

Couturier, É., Boyer, F., Pouliquen, O. \& Guazzelli, E. 2011 Suspensions in a tilted trough: second normal stress difference. Journal of Fluid Mechanics 10, 26-39.

Dai, S., Bertevas, E., QI, F. \& Tanner, R. 2013 Viscometric functions for noncolloidal sphere suspensions with newtonian matrices. Journal of Rheology 57 (2), 493-510.

Davit, Y. \& PEYla, P. 2008 Intriguing viscosity effects in confined suspensions: A numerical study. EPL (Europhysics Letters) 83 (6), 64001.

Dbouk, T., Lobry, L. \& Lemaire, E. 2013 Normal stresses in concentrated non-brownian suspensions. Journal of Fluid Mechanics 715, 239-272.

Eral, H.B., van den Ende, D., Mugele, F. \& Duits, M.H. 2009 Influence of confinement by smooth and rough walls on particle dynamics in dense hard-sphere suspensions. Physical Review E 80 (6), 061403.

Fernandez, N., Mani, R., Rinaldi, D., Kadau, D., Mosquet, M., Lombois-Burger, H., Cayer-Barrioz, J., Herrmann, H., Spencer, N. \& Isa, L. 2013 Microscopic mechanism for shear thickening of non-brownian suspensions. Physical review letters 111 (10), 108301.

Gallier, S. 2014 Simulation numérique de suspensions frictionnelles. Application aux propergols solides. PhD thesis, Université de Nice-Sophia Antipolis.

Gallier, S., Lemaire, E., Lobry, L. \& Peters, F. 2014a A fictitious domain approach for the simulation of dense suspensions. Journal of Computational Physics 256, 367-387.

Gallier, S., Lemaire, E., Peters, F. \& Lobry, L. $2014 b$ Rheology of sheared suspensions of rough frictional particles. Journal of Fluid Mechanics 757, 514-549.

Gamonpilas, C., Morris, J.F. \& Denn, M.M. 2016 Shear and normal stress measurements in non-brownian monodisperse and bidisperse suspensions. Journal of Rheology (1978-present) 60 (2), 289-296.

Ganatos, P., Weinbaum, S. \& Pfeffer, R. 1982 Gravitational and zero-drag motion of a sphere of arbitrary size in an inclined channel at low reynolds number. Journal of Fluid Mechanics 124, 27-43.

Garland, S, Gauthier, G, Martin, J \& Morris, JF 2013 Normal stress measurements in sheared non-brownian suspensions. Journal of Rheology (1978-present) 57 (1), 71-88.

JANA, S.C., KAPOOR, B. \& ACRIvos, A. 1995 Apparent wall slip velocity coefficients in concentrated suspensions of noncolloidal particles. Journal of Rheology (1978-present) 39 (6), $1123-1132$.

JEFFrEY, D.J. 1992 The calculation of the low Reynolds number resistance functions for two unequal spheres. Physics of Fluids A: Fluid Dynamics 4, 16. 
Jeffrey, D.J., Morris, J.F. \& Brady, J..F 1993 The pressure moments for two rigid spheres in low-reynolds-number flow. Physics of Fluids A 5 (10), 2317-2325.

Kim, S. \& KARRILA, S.J. 1991 Microhydrodynamics: principles and selected applications, , vol. 507. Butterworth-Heinemann Boston.

Kromkamp, J., van den Ende, D., Kandhai, D., van der Sman, R. \& Boom, R. 2006 Lattice boltzmann simulation of $2 \mathrm{~d}$ and $3 \mathrm{~d}$ non-brownian suspensions in couette flow. Chemical engineering science $\mathbf{6 1}(2), 858-873$.

KulKarni, S.D. \& MorRis, J.F. 2009 Ordering transition and structural evolution under shear in brownian suspensions. Journal of Rheology $\mathbf{5 3}$ (2), 417-439.

Lootens, D., Van Damme, H., Hémar, Y. \& HÉBraud, P. 2005 Dilatant flow of concentrated suspensions of rough particles. Physical review letters 95 (26), 268302.

Mari, R., Seto, R., Morris, J.F. \& Denn, M.M. 2014 Shear thickening, frictionless and frictional rheologies in non-brownian suspensions. Journal of Rheology (1978-present) 58 (6), 1693-1724.

Metzger, B., Rahli, O. \& Yin, X. 2013 Heat transfer across sheared suspensions: role of the shear-induced diffusion. Journal of Fluid Mechanics 724, 527-552.

Michailidou, V.N., Petekidis, G., Swan, J.W. \& Brady, J.F. 2009 Dynamics of concentrated hard-sphere colloids near a wall. Physical review letters 102 (6), 068302.

MorRis, J.F. 2009 A review of microstructure in concentrated suspensions and its implications for rheology and bulk flow. Rheologica acta 48 (8), 909-923.

NGuyen, N.Q. \& LAdD, AJC 2002 Lubrication corrections for lattice-Boltzmann simulations of particle suspensions. Physical Review E 66 (4), 046708.

Peyla, P. \& Verdier, C. 2011 New confinement effects on the viscosity of suspensions. EPL (Europhysics Letters) 94 (4), 44001.

Pieper, S. \& SCHMid, H. 2016 Layer-formation of non-colloidal suspensions in a parallel plate rheometer under steady shear. Journal of Non-Newtonian Fluid Mechanics pp. 1-7.

Rintoul, M.D. \& Torquato, S. 1996 Computer simulations of dense hard-sphere systems. The Journal of chemical physics 105 (20), 9258-9265.

Royer, J.R., Blair, D.L. \& Hudson, S.D. 2016 A rheological signature of frictional interactions in shear thickening suspensions. arXiv preprint arXiv:1603.00422 .

Sangani, A., Acrivos, A. \& Peyla, P. 2011 Roles of particle-wall and particle-particle interactions in highly confined suspensions of spherical particles being sheared at low reynolds numbers. Physics of Fluids 23, 083302.

Seto, R., Mari, R., Morris, J.F. \& Denn, M.M. 2013 Discontinuous shear thickening of frictional hard-sphere suspensions. Physical review letters 111 (21), 218301.

ShäFer, J, Dippel, S \& Wolf, DE 1996 Force schemes in simulations of granular materials. Journal de physique I 6 (1), 5-20.

Sierou, A. \& Brady, J.F. 2001 Accelerated stokesian dynamics simulations. Journal of Fluid Mechanics 448, 115-146.

Sierou, A. \& Brady, J.F. 2002 Rheology and microstructure in concentrated noncolloidal suspensions. Journal of Rheology 46, 1031.

Silbert, L.E., Ertaş, D., Grest, G.S., Halsey, T.C., Levine, D. \& Plimpton, S.J. 2001 Granular flow down an inclined plane: Bagnold scaling and rheology. Phys. Rev. E 64 (5), 051302 .

Singh, A. \& Nott, P.R. 2000 Normal stresses and microstructure in bounded sheared suspensions via Stokesian dynamics simulations. Journal of Fluid Mechanics 412, 279-301.

Singh, A. \& NotT, P.R. 2003 Experimental measurements of the normal stresses in sheared stokesian suspensions. Journal of Fluid Mechanics 490, 293-320.

Smart, J.R. \& Leighton, D.T. 1989 Measurement of the hydrodynamic surface roughness of noncolloidal spheres. Physics of Fluids A: Fluid Dynamics 1 (1), 52-60.

Snook, B., Butler, J.E. \& Guazzelli, É. 2015 Dynamics of shear-induced migration of spherical particles in oscillatory pipe flow. Journal of Fluid Mechanics 786, 128-153.

Stickel, J.J. \& Powell, R.L. 2005 Fluid mechanics and rheology of dense suspensions. Annual Review of Fluid Mechanics 37, 129-149.

Volkov, I., Cieplak, M., Koplik, J. \& Banavar, J. 2002 Molecular dynamics simulations of crystallization of hard spheres. Physical Review E 66 (6), 061401. 
Yeo, K. \& MAXey, M.R. $2010 a$ Anomalous diffusion of wall-bounded non-colloidal suspensions in a steady shear flow. EPL (Europhysics Letters) 92 (2), 24008.

YeO, K. \& MAXEY, M.R. $2010 b$ Dynamics of concentrated suspensions of non-colloidal particles in couette flow. Journal of Fluid Mechanics 649 (1), 205-231.

YeO, K. \& MAXEY, M.R. 2010c Ordering transition of non-brownian suspensions in confined steady shear flow. Physical Review E 81 (5), 051502.

Zarraga, I.E., Hill, D.A. \& Leighton JR, D.T. 2000 The characterization of the total stress of concentrated suspensions of noncolloidal spheres in newtonian fluids. Journal of Rheology 44, 185.

Zurita-Gotor, M., BŁawzdziewicz, J. \& Wajnryb, E. 2007 Swapping trajectories: A new wall-induced cross-streamline particle migration mechanism in a dilute suspension of spheres. Journal of Fluid Mechanics 592, 447-469. 